\title{
Potential of Cameroonian Plants and Derived Products against Microbial Infections: A Review
}

Author

Affiliation
Victor Kuete

Department of Biochemistry, University of Dschang, Dschang, Cameroon
Key words

- infectious diseases

- bacteria

- fungi

- medicinal plants

- bioactive compounds

- Cameroon received February 8, 2010

revised April 29, 2010

accepted May 5, 2010

Bibliography

Dol http://dx.doi.org/

10.1055/s-0030-1250027

Published online June 8, 2010

Planta Med 2010; 76:

1479-1491 @ Georg Thieme

Verlag KG Stuttgart · New York .

ISSN 0032-0943

Correspondence

\section{Dr. Victor Kuete}

Department of Biochemistry

University of Dschang

P. O. Box 67

Dschang 237

Cameroon

Phone: +23777355927

Fax: +2372226018

kuetevictor@yahoo.fr

\section{Abstract \\ $\nabla$}

In Cameroon, infectious diseases are amongst the most commonly notified diseases and largest cause of mortality. Many plants are used locally in traditional medicine for their treatment. The aim of the present review is to summarize currently available evidence and knowledge concerning Cameroonian plants used to treat bacterial and fungal infections, and the efficacy of plantderived extracts and compounds. The traditional uses of plants in the treatment of infectious diseases have been collected and tabulated. The antimicrobial activity of the extracts and the chemical constituents of most of these plants are summarized in this report. Plants used traditionally in Ca-

\section{Introduction}

$\nabla$

The importance of medicinal plants as a source of new antimicrobials is well established today. Approximately $25 \%$ of the active substance prescriptions in the United States come from plant material [1]. It is estimated that as many as 20000 species from several families are useful for these purposes [2]. In the two last decades, the search for antimicrobial potential of medicinal plants in Cameroon has experienced a tremendous growth. Significant numbers of scientific publications have been produced and many research teams have addressed this area. When searchingfor publications relative to the antimicrobial activity of Cameroonian medicinal plants or compounds from natural sources, it was found that the first works were published in 1988 by Biyiti et al. [3]. Evidence of the efficiency of herbal drugs used in Cameroonian medicine in the treatment of microbial infections is being provided continuously and intensively today [4-6]. More than a hundred studies were published from 1987 to 2009, according to scientific websites such as Pubmed, meroonian medicine, with laboratory work on any part or products, have been documented. Numerous extracts and compounds have been tested for antimycobacterial, antibacterial and antifungal efficacy and some of them were significantly active. Most of the bioactive compounds isolated were phenolics and alkaloids. In conclusion, many plant species are used in traditional medicine in Cameroon to treat infectious diseases, and several interesting openings have originated for further inquiry following in vitro antimicrobial activity evaluation. However, much work is still to be done to standardize methods and cut-off points for describing the antimicrobial activity, and on the study of the mechanisms of action.

Sciencedirect, Scirus and Scopus. This paper summarizes the currently available knowledge on Cameroonian plants used to treat microbial diseases, and the efficacy of plant-derived extracts and compounds. Numerous medicinal plants are commonly used in both urban and rural areas in Cameroon as well as in most African countries, in the treatment of infectious diseases. The WHO [7] estimated that $80 \%$ of the African population is concerned and believes that this practice, if applied appropriately, could significantly contribute to improve public health. In the plant kingdom, medicinal plants from several families are used for therapeutic purposes. This review will be focused on the plant families Moraceae, Irvingiaceae, Melianthaceae, Rutaceae, Guttiferae, Bignogniaceae, Ebenaceae, etc., reported for their antimicrobial activities. Several classes of secondary metabolites have been characterized as the active principles of the plants, including terpenoids, alkaloids and phenolic compounds such as chalcones, flavones, isoflavones, anthraquinones, naphthoquinones, xanthones, and coumarins. Later in this report, we will discuss the use of the 
Table 1 Alphabetic list of the microbial species.

\begin{tabular}{|c|c|c|c|c|c|}
\hline Microorganisms & Abbreviation & Microorganisms & Abbreviation & Microorganisms & Abbreviation \\
\hline Aspergillus niger & A. niger & Cladosporium sp. & - & Salmonella typhi & S. typhi \\
\hline Aspergillus flavus & A. flavus & Enterococcus hirae & E. hirae & Pseudomonas aeruginosa & P. aeruginosa \\
\hline Alternaria sp. & - & Escherichia coli & E. coli & Scenedesmus subspicatus & S. subspicatus \\
\hline Bacillus subtilis & B. subtilis & Fusarium sp. & - & Shigella dysenteriae & S. dysenteriae \\
\hline $\begin{array}{l}\text { Bacillus stearothermophi- } \\
\text { lus }\end{array}$ & B. stearothermophilus & Geotrichum candidum & G. candidum & Shigella flexneri & S. flexneri \\
\hline Bacillus cereus & B. cereus & Klebsiella pneumoniae & K. pneumoniae & Staphylococcus aureus & S. aureus \\
\hline Bacillus megaterium & B. megaterium & Microsporum audouinii & M. audouinii & $\begin{array}{l}\text { Staphylococcus saprophy- } \\
\text { ticus }\end{array}$ & S. saprophyticus \\
\hline Aspergillus ochraceus & A. ochraceus & Citrobacter freundii & C. freundii & Streptococcus anginosus & S. anginosus \\
\hline Enterobacter cloacae & E. cloacae & $\begin{array}{l}\text { Cryptococcus neofor- } \\
\text { mans }\end{array}$ & C. neoformans & Streptococcus faecalis & S. faecalis \\
\hline Enterobacter aerogenes & E. aerogenes & Morganella morganii & M. morganii & Streptococcus mutans & S. mutans \\
\hline Candida glabrata & C. glabrata & Mucor miehei & M. miehei & Streptococcus oralis & S. oralis \\
\hline $\begin{array}{l}\text { Corynebacterium glutami- } \\
\text { cum }\end{array}$ & C. glutamicum & $\begin{array}{l}\text { Mycobacterium smeg- } \\
\text { matis }\end{array}$ & M. smegmatis & Streptococcus pneumoniae & S. pneumoniae \\
\hline Candida albicans & C. albicans & $\begin{array}{l}\text { Mycobacterium tuber- } \\
\text { culosis }\end{array}$ & M. tuberculosis & Streptococcus pneumoniae & S. pneumoniae \\
\hline Haemophilus influenza & H. influenza & Neisseria gonorrhoeae & N. gonorrhoeae & Streptococcus pyogenes & S. pyogenes \\
\hline Chlorella sorokiniana & C. sorokiniana & Penicillium sp. & - & $\begin{array}{l}\text { Streptomyces viridochro- } \\
\text { mogenes }\end{array}$ & S. viridochromogenes \\
\hline Candida krusei & C. krusei & Penicillium verrucosum & P. verrucosum & $\begin{array}{l}\text { Trichophyton mentago- } \\
\text { phytes }\end{array}$ & T. mentagophytes \\
\hline Candida tropicalis & C. tropicalis & Proteus mirabilis & P. mirabilis & Trichophyton rubrum & T. rubrum \\
\hline Chlorella vulgaris & C. vulgaris & Proteus vulgaris & P. vulgaris & Vibrio anguillarium & V. anguillarium \\
\hline
\end{tabular}

(-) Only provided when the species is determined

studied plants in traditional therapy, the antimicrobial activity of extracts of the plants studied in each family, and finally the plantderived metabolites characterized to date in Cameroon.

\section{Impact of Infectious Disease Worldwide and in Cameroon}

$\nabla$

With the advent of globalization, health threats have become much more serious in an increasingly interconnected world, characterized by higher mobility of people, animals and goods, economic interdependence and electronic connectivity [8]. According to the WHO, at least 39 new pathogens have been identified since 1967, including HIV, Ebola and Marburg hemorrhagic fevers [9]. In addition, "centuries-old threats" like influenza, malaria and tuberculosis continue to thrive due to a combination of biological mutations, rising resistance to antibiotics and weak health systems [9]. In the last five years, the WHO has verified more than 1100 epidemic events worldwide [8]. Infectious diseases cause about $70 \%$ of deaths in children in developing countries and more than a third of those deaths occur in neonates [8]. More than $80 \%$ of tuberculosis cases occur in Asia and Africa [10]. In Cameroon, the major infectious diseases associated with a high degree of risk within the population include food or waterborne diseases (bacterial and protozoal diarrhea, hepatitis A and E, and typhoid fever), vector borne diseases (malaria and yellow fever), water contact disease (schistosomiasis), respiratory disease (meningococcal meningitis), and animal contact disease (rabies) [11]. Very often, there is a coexistence of many infectious diseases. Ammah et al. [12] demonstrated that a high proportion of patients (33\%) had malaria coexisting with S. typhimurium, S. paratyphi, and S. typhi infections. In our population, the lifetime risk of developing active tuberculosis once infected, in the absence of
HIV infection, is about $10 \%$, meanwhile this risk increases tenfold in HIV-infected individuals [13]. The unsatisfactory case management of the whole infectious diseases in general, and particularly bacterial and fungal infections throughout the continent, which allows partially treated and relapsed patients to become sequentially resistant, may play a significant role in the development of resistance $[14,15]$. Effective treatment of microbial infections is challenging for various reasons including lack of accessibility and elevated expense of drugs and low adherence owing to toxicity of second-line drugs $[14,15]$. It is all too likely that the emergence of even more resistant microbial strains will be experienced in the future, exhausting the current arsenal of chemical defenses at our disposal [14]. For this purpose, new antimicrobial agents are urgently needed, and research programs into alternative therapeutics should be encouraged. It has been suggested that the best available in vitro indicator of possible therapeutic activity is the early microbicidal activity of medicinal plants [7], drugs or combinations of drugs [16].

\section{Investigation of Plants and Derived Products as Sources of New Antimicrobial Agents \\ $\nabla$}

Plants produce a great diversity of substances that could be active in many fields of medicine. Natural products from plants are proven templates for new drug development [17], and have shown many interesting biological activities. In a review of medicinal plants as antimicrobial agents [18], it was estimated that at least 12000 active compounds have been isolated from plants, representing less than $10 \%$ of the total. Several recent reviews have highlighted the underutilized potential of plant species and natural products as sources of antimicrobial drugs [14]. Plant-derived antimicrobial compounds belong to an exceptionally wide 
Table 2 Plants used in Cameroon to treat infectious diseases, with evidence of their activities.

\begin{tabular}{|c|c|c|c|c|c|}
\hline Family & Species $^{\mathrm{a}}$ & Traditional treatment & $\begin{array}{l}\text { Plant part } \\
\text { used }\end{array}$ & Bioactive (or potentially active) compounds & $\begin{array}{l}\text { Screened activityb for } \\
\text { crude plant extract }\end{array}$ \\
\hline \multirow[t]{2}{*}{$\begin{array}{l}\text { Annona- } \\
\text { ceae }\end{array}$} & $\begin{array}{l}\text { Monodora } \\
\text { myristica }\end{array}$ & $\begin{array}{l}\text { headache, constipation, } \\
\text { sores, guinea worm } \\
\text { infections [77] }\end{array}$ & seeds & $\begin{array}{l}\text { not identified but the active essential oil from fruits } \\
\text { contained } \alpha \text {-phellandrene; } p \text {-cymene; } \alpha \text {-pinene; cis- } \\
\text { sabinol; limonene [77] }\end{array}$ & $\begin{array}{l}\text { (fruits essential oils) Q: } A f \text {, } \\
B c, B s, C g l, E c, K p, S a, S f[77]\end{array}$ \\
\hline & $\begin{array}{l}\text { Xylopia } \\
\text { aethiopica }\end{array}$ & $\begin{array}{l}\text { cough, bronchitis, } \\
\text { dysentery, female } \\
\text { sterility [77] }\end{array}$ & seeds & $\begin{array}{l}\text { not identified but the active essential oil from fruits } \\
\text { contained } \beta \text {-pinene; terpinen- } 4 \text {-ol; sabinene; } \alpha \text {-phel- } \\
\text { landrene; } \alpha \text {-terpineol; } \alpha \text { - and trans- } \beta \text {-ocimene [77] }\end{array}$ & $\begin{array}{l}\text { (fruits essential oils) S: Af, } \\
B c, E c, S a, S f ; \mathbf{Q}: C g l[77]\end{array}$ \\
\hline $\begin{array}{l}\text { Apocyna- } \\
\text { ceae }\end{array}$ & $\begin{array}{l}\text { Tabernaemon- } \\
\text { tana crassa } \\
\text { Benth. } \\
(43449 / \mathrm{HNC})\end{array}$ & $\begin{array}{l}\text { gonorrhea fungal infec- } \\
\text { tions, ovarian trouble, } \\
\text { anthrax, headache, con- } \\
\text { stipation, disinfections, } \\
\text { homeostasis [78] }\end{array}$ & $\begin{array}{l}\text { leaves, } \\
\text { stem bark, } \\
\text { sap }\end{array}$ & $\begin{array}{l}\text { dehydrocorydalmine; palmatine; isoursenol; ace- } \\
\text { tate of isoursenol; lupeol [76] }\end{array}$ & $\begin{array}{l}\text { (bark methanol extract) W: } \\
\text { Ca, Ck, Ec, Kp, Ng, Pa, Pv, Sa, } \\
\text { Sd, Sf, Sp, St [76] }\end{array}$ \\
\hline \multirow[t]{2}{*}{ Asteraceae } & $\begin{array}{l}\text { Emilia coccinea } \\
\text { (Sims) G. Don } \\
\text { (6297/Leeu- } \\
\text { wenberg) }\end{array}$ & $\begin{array}{l}\text { diarrhea, stomachache, } \\
\text { bowel, bladder disor- } \\
\text { ders, wounds disinfec- } \\
\text { tion [79] }\end{array}$ & leaves & $\begin{array}{l}\text { not identified but preliminary phytochemical study } \\
\text { of active methanol leaf extract revealed the pres- } \\
\text { ence of alkaloids, flavonoids, tannins, saponins and } \\
\text { cardiac glycosides [80] }\end{array}$ & $\begin{array}{l}\text { (leaves methanol extract) } \\
\mathbf{W : E c}, S a, S t[80]\end{array}$ \\
\hline & $\begin{array}{l}\text { Crepis came- } \\
\text { roonica Babc. }\end{array}$ & $\begin{array}{l}\text { diarrhea, wounds and } \\
\text { fungal infections [81] }\end{array}$ & $\begin{array}{l}\text { not speci- } \\
\text { fied }\end{array}$ & 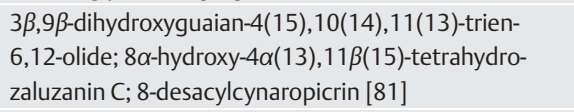 & $\begin{array}{l}\text { (aerial part methanol } \\
\text { extract) Q: Ec, Sa [81] }\end{array}$ \\
\hline \multirow[t]{2}{*}{$\begin{array}{l}\text { Bignognia- } \\
\text { ceae }\end{array}$} & $\begin{array}{l}\text { Newbouldia } \\
\text { laevis Seem. } \\
\text { (1754/SRFK) }\end{array}$ & $\begin{array}{l}\text { diarrhea, dysentery, } \\
\text { worms, malaria, sexu- } \\
\text { ally transmitted dis- } \\
\text { eases, dental caries [83] }\end{array}$ & $\begin{array}{l}\text { leaves, } \\
\text { stem bark, } \\
\text { roots }\end{array}$ & $\begin{array}{l}\text { newbouldiaquinone A [82]; chrysoeriol; newboul- } \\
\text { diaquinone; 2-acetylfuro-1,4-naphthoquinone; } 2 \text { - } \\
\text { hydroxy-3-methoxy-9,10-dioxo-9,10-dihydroanthra- } \\
\text { cene-1-carbaldehyde; lapachol; } \beta \text {-sitosterol-3-O- } \beta \text { - } \\
\text { dglucopyranoside; oleanolic acid; canthic acid; } \\
\text { newbouldiamide; } 2 \text {-(4-hydroxyphenyl)-ethyl triocta- } \\
\text { noate [48] }\end{array}$ & $\begin{array}{l}\text { (bark methanol extract) S: } \\
B c, B m, B s, B s t ; \text { M: Ca, Ck, } \\
\mathrm{Cg}, \mathrm{Ea}, \mathrm{Ec} \mathrm{Ecl}, \mathrm{Cf}, \mathrm{Mm}, \mathrm{Kp} \text {, } \\
\text { Pa, Pm, Pv, Sd, Sfl, Sf, St; } \\
\text { W: Sa [48] }\end{array}$ \\
\hline & $\begin{array}{l}\text { Stereosper- } \\
\text { mum zenkeri } \\
\text { K. Schum. ex } \\
\text { De Wild } \\
\text { (1022/SRFK) }\end{array}$ & $\begin{array}{l}\text { bronchitis, microbial in- } \\
\text { fections [84] }\end{array}$ & $\begin{array}{l}\text { leaves, } \\
\text { stem bark }\end{array}$ & $\begin{array}{l}\text { zenkequinone } A \text { and } B \text {, sterequinone-F, } p \text {-coumaric } \\
\text { acid [84] }\end{array}$ & $\begin{array}{l}\text { crude extract was not in- } \\
\text { vestigated but zenkequi- } \\
\text { none B presented a MIC } \\
\text { value of } 9.50 \mu \mathrm{g} / \mathrm{mL} \text { on } \\
\text { P. aeruginosa [84] }\end{array}$ \\
\hline $\begin{array}{l}\text { Caesalpi- } \\
\text { niaceae }\end{array}$ & $\begin{array}{l}\text { Erythrophleum } \\
\text { suaveolens } \\
\text { (Guill. \& Perr.) } \\
\text { Brenan, } \\
\text { (2644/SRFK) }\end{array}$ & $\begin{array}{l}\text { inflammation, analgesic, } \\
\text { bacterial and fungal infec- } \\
\text { tions, chickenpox, gangre- } \\
\text { nous sores, cardiovascular } \\
\text { diseases [85] }\end{array}$ & stem bark & norcassaïde; norerythrosuaveolide [75] & $\begin{array}{l}\text { crude extract was not in- } \\
\text { vestigated but active diter- } \\
\text { penoid alkaloids were iso- } \\
\text { lated from the stem bark } \\
\text { [75] }\end{array}$ \\
\hline \multirow[t]{2}{*}{ Ebenaceae } & $\begin{array}{l}\text { Diospyros } \\
\text { crassiflora } \\
\text { (4924/SRFK) }\end{array}$ & $\begin{array}{l}\text { gonorrhea and other } \\
\text { bacterial and fungal in- } \\
\text { fections, tuberculosis } \\
{[49,86-88]}\end{array}$ & stem bark & crassiflorone; diospyrone; plumbagin $[49,86-88]$ & $\begin{array}{l}\text { (bark dichloromethane: } \\
\text { methanol } 1: 1 \text { extract) W: } \\
\text { An, } A f, A s p, C a, C g, C k, C t, \\
\text { Csp, Cn, Fsp, Gc, Psp [88] } \\
\text { (Bark methanol extract) S: } \\
\text { Ms, Mtb, Ng [49] }\end{array}$ \\
\hline & $\begin{array}{l}\text { Diospyros } \\
\text { canaliculata } \\
\text { (9653/SRF/ } \\
\text { cam) }\end{array}$ & $\begin{array}{l}\text { gonorrhea and other } \\
\text { bacterial and fungal in- } \\
\text { fections, tuberculosis } \\
{[49,86]}\end{array}$ & stem bark & diospyrone; plumbagin $[49,86]$ & S: Ms, Mtb, Ng [49] \\
\hline \multirow[t]{3}{*}{$\begin{array}{l}\text { Euphorbia- } \\
\text { ceae }\end{array}$} & $\begin{array}{l}\text { Bridelia grandis } \\
\text { Pierre ex } \\
\text { Hutch } \\
\text { (BWPV01) }\end{array}$ & $\begin{array}{l}\text { rheumatism, arthritis, } \\
\text { abdominal pain, thrush, } \\
\text { oral cavity affection [89, } \\
\text { 90] }\end{array}$ & $\begin{array}{l}\text { stem bark, } \\
\text { leaves, } \\
\text { roots, } \\
\text { fruits }\end{array}$ & $\begin{array}{l}\text { not identified but qualitative phytochemical analyses } \\
\text { and colorimetric assays, together with preliminary } \\
\text { chromatographic separations of the most active bark } \\
\text { extracts, clearly suggested the presence of condensed } \\
\text { tannins as main constituents of the phytocomplex re- } \\
\text { sponsible for the biological activity [90] }\end{array}$ & $\begin{array}{l}\text { (bark water extract) M: Sm, } \\
\text { San, So, Sp [90] }\end{array}$ \\
\hline & $\begin{array}{l}\text { Bridelia } \\
\text { ferruginea }\end{array}$ & $\begin{array}{l}\text { dysentery, diabetes, } \\
\text { thrush mycotic stomati- } \\
\text { tis in children, antidote } \\
\text { for snakebite, gonor- } \\
\text { rhea, poisons [91] }\end{array}$ & $\begin{array}{l}\text { stem bark, } \\
\text { leaves, } \\
\text { fruits }\end{array}$ & $\begin{array}{l}\text { not identified but qualitative phytochemical analy- } \\
\text { ses of the plant revealed the presence of triter- } \\
\text { penes, steroids, tannins, saponins, flavonoids [92] }\end{array}$ & $\begin{array}{l}\text { (leave methanol extract) } \\
\text { W: } B s, E c, S a, S f[92]\end{array}$ \\
\hline & $\begin{array}{l}\text { Mallotus } \\
\text { oppositifolium }\end{array}$ & $\begin{array}{l}\text { diarrhea and dysentery } \\
\text { [93] }\end{array}$ & leaves & not yet identified & $\begin{array}{l}\text { (leave methanol extract) } \\
\text { W: } S d[93]\end{array}$ \\
\hline Fabaceae & $\begin{array}{l}\text { Eriosema } \\
\text { glomerata } \\
643 / \mathrm{HNC}\end{array}$ & infectious diseases [94] & $\begin{array}{l}\text { whole } \\
\text { plant }\end{array}$ & $\begin{array}{l}\text { erioschalcones A, erioschalcones B, quercetin, iso- } \\
\text { luteolin [94] }\end{array}$ & $\begin{array}{l}\text { Crude extract was not in- } \\
\text { vestigated but active com- } \\
\text { pounds were isolated from } \\
\mathrm{CH}_{2} \mathrm{Cl}_{2}-\mathrm{MeOH} \text { extract of } \\
\text { the whole plant [94] }\end{array}$ \\
\hline
\end{tabular}


Table 2 Plants used in Cameroon to treat infectious diseases, with evidence of their activities. (continued)

\begin{tabular}{|c|c|c|c|c|c|}
\hline Family & Species $^{a}$ & Traditional treatment & $\begin{array}{l}\text { Plant part } \\
\text { used }\end{array}$ & Bioactive (or potentially active) compounds & $\begin{array}{l}\text { Screened activityb for } \\
\text { crude plant extract }\end{array}$ \\
\hline \multirow[t]{12}{*}{ Guttiferae } & $\begin{array}{l}\text { Mammea } \\
\text { Africana } 4221 / \\
\text { SRF/CAM }\end{array}$ & $\begin{array}{l}\text { stomach pains, scabies, } \\
\text { skin diseases, rheumatic } \\
\text { pain, cough [95] }\end{array}$ & $\begin{array}{l}\text { stem bark, } \\
\text { fruit }\end{array}$ & mammea A/AA, mammea C/OB [95] & $\begin{array}{l}\text { crude extract was not } \\
\text { tested but active couma- } \\
\text { rins were isolated from the } \\
\text { stem bark [95] }\end{array}$ \\
\hline & $\begin{array}{l}\text { Allablackia } \\
\text { gabonensis } \\
\text { (Pellegr.) } \\
\text { Bamps } \\
(23255 / \text { HNC) }\end{array}$ & $\begin{array}{l}\text { dysenteries, cold, tooth } \\
\text { aches [56] }\end{array}$ & stem bark & $\begin{array}{l}\text { allanxanthone A; allanxanthone D; 1,3,6,7-tetrahy- } \\
\text { droxy-2-(3-methylbut-2-enyl)xanthone [56] }\end{array}$ & $\begin{array}{l}\text { crude extract was not in- } \\
\text { vestigated but active xan- } \\
\text { thones were isolated from } \\
\text { the stem bark [56] }\end{array}$ \\
\hline & $\begin{array}{l}\text { Calophyllum } \\
\text { inophyllum L. } \\
\text { (32189/SRF/ } \\
\text { Cam) }\end{array}$ & $\begin{array}{l}\text { cicatrisant,analgesic, } \\
\text { wounds and herpes in- } \\
\text { fections [74] }\end{array}$ & $\begin{array}{l}\text { stem bark, } \\
\text { roots, } \\
\text { fruits }\end{array}$ & $\begin{array}{l}\text { caloxanthone A, calophynic acid, brasiliensic acid, } \\
\text { inophylloidic acid, calaustralin, calophyllolide, ino- } \\
\text { phyllum C, inophyllum E [96] }\end{array}$ & $\begin{array}{l}\text { [(root bark and fruits } \\
\mathrm{CH}_{2} \mathrm{Cl}_{2}-\mathrm{MeOH}(1: 1) \text { ex- } \\
\text { tract] Q: } \mathrm{Sa}[96]\end{array}$ \\
\hline & $\begin{array}{l}\text { Garcinia kola } \\
\text { Heckel }\end{array}$ & $\begin{array}{l}\text { infectious diseases, res- } \\
\text { piratory tract infections } \\
{[97]}\end{array}$ & fruits & not yet identified & $\begin{array}{l}\text { (fruits ethanol extract) } \\
\text { S: Sa, Sp, Spn, } H i[97]\end{array}$ \\
\hline & $\begin{array}{l}\text { Garcinia } \\
\text { smeathmanii } \\
\text { oliver ( } 35169 \text { / } \\
\text { HNC) }\end{array}$ & $\begin{array}{l}\text { bacterial and fungal in- } \\
\text { fections [51] }\end{array}$ & stem bark & $\begin{array}{l}\text { cheffouxanthone; } 1,5 \text { dihydroxyxanthone; } 1,3,5 \text {-tri- } \\
\text { hydroxyxanthone; bangangxanthone A; smeath- } \\
\text { xanthone B; smeathxanthone A; guttiferone I; iso- } \\
\text { xanthochymol; friedelin; triacontanyl caffeate [51] }\end{array}$ & $\begin{array}{l}\text { (bark methanol extract) } \\
\text { S: Bm, Bs, Ea, Ec, Kp, Mm, Pa, } \\
P v, S f, S t ; \mathbf{M}: B c, B s t, C a, C g, \\
C k, C f, E c l, P m, S d[51]\end{array}$ \\
\hline & $\begin{array}{l}\text { Garcinia } \\
\text { staudtii Engl } \\
(167341 / \\
\text { HNC) }\end{array}$ & $\begin{array}{l}\text { bacterial infections, } \\
\text { cancer [98] }\end{array}$ & stem bark & $\begin{array}{l}\text { staudtiixanthone A; staudtiixanthone B (2); } \alpha \text {-man- } \\
\text { gostin; gartanin; staudtixanthones C; staudtiixan- } \\
\text { thones D; demethylcalabaxanthone garcinone B } \\
{[98]}\end{array}$ & $\begin{array}{l}\text { crude extract was not in- } \\
\text { vestigated but isolated } \\
\text { compounds were active on } \\
\text { S. aureus [98] }\end{array}$ \\
\hline & $\begin{array}{l}\text { Symphonia } \\
\text { globulifera } \\
\text { Linn f. (syn. S. } \\
\text { gabonensis } \\
\text { Pierre) (2235/ } \\
\text { SRFK) }\end{array}$ & $\begin{array}{l}\text { laxative for pregnant } \\
\text { women, fatigue, bacte- } \\
\text { rial infections }[56,99 \text {, } \\
100]\end{array}$ & $\begin{array}{l}\text { stem bark, } \\
\text { fruits }\end{array}$ & $\begin{array}{l}\text { globuliferin [100]; globulixanthone C; globulixan- } \\
\text { thone D; globulixanthone E [56] }\end{array}$ & $\begin{array}{l}\text { (seeds methanol extract) } \\
\mathbf{W}: E c, S a, S f, K p[100]\end{array}$ \\
\hline & & & & & $\begin{array}{l}\text { (roots bark } \mathrm{CH}_{2} \mathrm{Cl}_{2}-\mathrm{MeOH} \\
\text { extract) } \mathrm{S}: \mathrm{Bs}, \mathrm{Sa}, \mathrm{Va}[56]\end{array}$ \\
\hline & $\begin{array}{l}\text { Vismia } \\
\text { guineensis } \\
\text { (Linn.) Choisy. } \\
\text { (75346/HNC) }\end{array}$ & $\begin{array}{l}\text { malaria, skin diseases, } \\
\text { bacterial infections [ } 53 \text {, } \\
\text { 101] }\end{array}$ & $\begin{array}{l}\text { leaves, } \\
\text { stem bark, } \\
\text { roots }\end{array}$ & $\begin{array}{l}\text { 3-geranyloxy-6-methyl-1,8-dihydroxyanthraqui- } \\
\text { none; vismiaquinone; vismiaquinone B; betulinic } \\
\text { acid (roots) [53]; vismiaquinone; caloxanthone ]; } \\
\text { O1-demethyl-3',4'-deoxypsorospermin-3',4'-diol; 6- } \\
\text { deoxyisojacareubin; 1,7-dihydroxyxanthone (barks) } \\
\text { [53]; friedelin; 1,8-dihydroxy-6-methoxy-3-methyl- } \\
\text { anthraquinone; kaempferol (leaves) [53] }\end{array}$ & $\begin{array}{l}\text { (leaves methanol extract) } \\
\text { S: Ca, Cf, Ecl, Mm, Sfl, Tm, Tr; } \\
\text { M: Bs, Bst, Cg, Ea, Ec, Kp, Ms, } \\
P m, P v, S a, S d, S t\end{array}$ \\
\hline & & & & & $\begin{array}{l}\text { (bark methanol extract) S: } \\
\text { Ca, Bst, Ecl, Mm, Sfl,Tm, Tr; } \\
\text { M: Bs, Ca, Cf, Cg, Ea, Ec, Kp, } \\
M s, P m, P v, S a, S d, S t\end{array}$ \\
\hline & & & & & $\begin{array}{l}\text { (roots methanol extract) S: } \\
\text { Ca, Bst, Ecl, Mm Ms, Mtb, Sfl, } \\
T m, T r ; \text { M: Bs, Ca, Cf, Cg, Ea, } \\
\text { Ec, Kp, Ms, Pm, Pv, Sa, Sd, St } \\
{[53]}\end{array}$ \\
\hline & $\begin{array}{l}\text { Vismia rubes- } \\
\text { cens Oliver } \\
\text { 43288/HNC }\end{array}$ & $\begin{array}{l}\text { skin diseases, diarrhea } \\
\text { and venereal diseases } \\
{[102]}\end{array}$ & $\begin{array}{l}\text { stem bark, } \\
\text { roots }\end{array}$ & $\begin{array}{l}\text { 1,4,8-trihydroxyxanthone; 1,7-dihydroxyxanthone; } \\
\text { physcion; friedelin; friedelanol [102] }\end{array}$ & $\begin{array}{l}\text { (bark methanol extract) } \\
\text { M: Sa, St; W: } P a, C a[102]\end{array}$ \\
\hline $\begin{array}{l}\text { Irvingia- } \\
\text { ceae }\end{array}$ & $\begin{array}{l}\text { Irvingia } \\
\text { gabonensis } \\
\text { (Aubry Le- } \\
\text { comte ex } \\
\text { O'Rorke) Baill. } \\
\text { (28054/HNC) }\end{array}$ & $\begin{array}{l}\text { gonorrhea, gastrointes- } \\
\text { tinal and hepatic dis- } \\
\text { orders, wound infec- } \\
\text { tions, diabetes, analge- } \\
\text { sic [103-107] }\end{array}$ & $\begin{array}{l}\text { leaves, } \\
\text { stem bark, } \\
\text { roots, } \\
\text { fruits }\end{array}$ & $\begin{array}{l}\text { 3-friedelanone; betulinic acid; oleanolic acid; 3,3,4- } \\
\text { tri-O-methylellagic acid; 3,4-di-O-methylellagic acid; } \\
\text { hardwickiic acid [39] }\end{array}$ & $\begin{array}{l}\text { (bark methanol extract) } \\
\text { S: Bst, } C a, C f, E a, E c l, M m, N g \text {, } \\
P a, P m, P v, S a, S d ; \mathbf{M}: B c, B m \text {, } \\
B s, C k, E c, K p, S f l, S t, S f[39]\end{array}$ \\
\hline \multirow[t]{2}{*}{ Lamiaceae } & $\begin{array}{l}\text { Ocimum } \\
\text { gratissimum }\end{array}$ & $\begin{array}{l}\text { pulmonary antiseptic, } \\
\text { antitussive, antispas- } \\
\text { modic [108] }\end{array}$ & leaves & $\begin{array}{l}\text { not identified but essential oils from fruits contained } \\
\text { thymol; } \gamma \text {-terpinene; } p \text {-cymene; limonene; } \alpha \text {-terpi- } \\
\text { nolene; } \alpha \text {-phellandrene; } 1,8 \text {-cineole; } \alpha \text {-terpineol; } \beta \text { - } \\
\text { caryophyllene; dehydro-p-cymene; } 3,9 \text {-epoxy-p- } \\
\text { mentha-1,8-diene [108] }\end{array}$ & $\begin{array}{l}\text { (essential oil) Q: Bc, Bs, Cgl, } \\
E c, S a, S f[102]\end{array}$ \\
\hline & $\begin{array}{l}\text { Thymus vulagris L } \\
42851 / \mathrm{HNC}\end{array}$ & fungal infections [109] & $\begin{array}{l}\text { whole } \\
\text { plant }\end{array}$ & essential oil, with nonidentified components [109] & (essential oil) Q: Ao [109] \\
\hline
\end{tabular}


Table 2 Plants used in Cameroon to treat infectious diseases, with evidence of their activities. (continued)

\begin{tabular}{|c|c|c|c|c|c|}
\hline Family & Species $^{a}$ & Traditional treatment & $\begin{array}{l}\text { Plant part } \\
\text { used }\end{array}$ & Bioactive (or potentially active) compounds & $\begin{array}{l}\text { Screened activity }{ }^{b} \text { for } \\
\text { crude plant extract }\end{array}$ \\
\hline Lauraceae & $\begin{array}{l}\text { Beilschmiedia } \\
\text { anacardioides } \\
\text { (Engl. \& K. } \\
\text { Krause) Robyns } \\
\text { \& Wilczek }\end{array}$ & $\begin{array}{l}\text { uterine tumors, Rubella, } \\
\text { female genital infec- } \\
\text { tions, rheumatism } \\
\text { [110] }\end{array}$ & stem bark & beilschmiedic acid A, B and C [110] & $\begin{array}{l}\text { crude extract was not in- } \\
\text { vestigated but active en- } \\
\text { diandric acid derivatives } \\
\text { were isolated from the } \\
\text { stem bark [110] }\end{array}$ \\
\hline \multirow[t]{11}{*}{ Moraceae } & $\begin{array}{l}\text { Dorstenia } \\
\text { angusticornis } \\
\text { Engl. (28165/ } \\
\text { SRFCam) }\end{array}$ & $\begin{array}{l}\text { gastroenteritis, diar- } \\
\text { rheal infections [42] }\end{array}$ & $\begin{array}{l}\text { whole } \\
\text { plant }\end{array}$ & $\begin{array}{l}\text { gancaonin Q; stipulin; angusticornin B; bartericin A } \\
\text { [42] }\end{array}$ & $\begin{array}{l}\text { (twigs methanol extract) } \\
\text { S: Bc, Bm, Ca, Ck, Ea, Ng, Pm, } \\
P v, S a, S d, S f, S f l ; M: B s, B s t, \\
C g, C f, E c, E c l, E c, K p, M m, P a, \\
\text { St [42] }\end{array}$ \\
\hline & $\begin{array}{l}\text { Dorstenia } \\
\text { barteri Bureau } \\
(44016 / \text { HNC) }\end{array}$ & $\begin{array}{l}\text { snakebite, rheumatic, } \\
\text { infectious diseases, ar- } \\
\text { thritis [111-113] }\end{array}$ & $\begin{array}{l}\text { whole } \\
\text { plant }\end{array}$ & $\begin{array}{l}\text { isobavachalcone; stipulin; 4-hydroxylonchocarpin; } \\
\text { kanzonol C; amentoflavone [5] }\end{array}$ & $\begin{array}{l}\text { (twigs methanol extract) } \\
\text { S: } B c, B m, B s, B s t, C a, C g, C f \\
C k, E a, E c, E c l, K p, M a, M m \\
P a, P m, P v, S a, S d, S f, S f l \\
\text { M: St, Tr [5] }\end{array}$ \\
\hline & $\begin{array}{l}\text { Dorstenia } \\
\text { elliptica } \\
\text { Bureau } \\
(44018 / \text { HNC) }\end{array}$ & eye infections [114] & $\begin{array}{l}\text { whole } \\
\text { plant }\end{array}$ & $\begin{array}{l}\text { psoralen; O-[3-(2,2-dimethyl-3-oxo-2H-furan-5-yl)- } \\
\text { butyl]bergaptol or dorstenin; bergapten; O-[3-(2,2- } \\
\text { dimethyl-3-oxo-2H-furan-5-yl)-3-hydroxybutyl]ber- } \\
\text { gaptol; 3-(3,3-dimethylallyl)-4,2',4'-trihydroxychal- } \\
\text { cone [43] }\end{array}$ & $\begin{array}{l}\text { (twigs methanol extract) } \\
\text { S: Bm, Bst, Ca, Cf, Ea, Ec, Ecl, } \\
P m, P v, S f, S f l ; \mathrm{M}: B c, B s, C g \text {, } \\
\text { Kp, Ma, Pa, Pv, Sa, St, Tr [43] }\end{array}$ \\
\hline & $\begin{array}{l}\text { Dorstenia } \\
\text { turbinata Engl. } \\
\text { (28158/SRF/ } \\
\text { Cam) }\end{array}$ & $\begin{array}{l}\text { gastroenteritis, skin in- } \\
\text { fections, gastroenteri- } \\
\text { tis, skin infections, rheu- } \\
\text { matism [6] }\end{array}$ & $\begin{array}{l}\text { whole } \\
\text { plant }\end{array}$ & $\begin{array}{l}\text { 5-methoxy-3-[3-( } \beta \text {-glucopyranosyloxy)-2-hydroxy-3- } \\
\text { methylbutyl]psoralen; 5-methoxy-3-(3-methyl-2,3- } \\
\text { dihydroxybutyl)psoralen; (2'S,3'R)-3'-hydroxymar- } \\
\text { mesin; 4-hydroxy-3-ethoxybenzaldehyde; 4-me- } \\
\text { thoxyphenol, psoralen; kanzonol C; 4-hydroxylon- } \\
\text { chocarpin; umbelliferone [6] }\end{array}$ & $\begin{array}{l}\text { (twigs methanol extract) } \\
\text { S: Ca, Cf, Cg, Ec, Kp, Ma, Pa, } \\
\text { Sa, Sd, St, Tr [6] }\end{array}$ \\
\hline & $\begin{array}{l}\text { Ficus chamy- } \\
\text { docarpa } \\
\text { Mildbraed \& } \\
\text { Burret. } \\
\text { (35446/HNC) }\end{array}$ & $\begin{array}{l}\text { filaris, diarrheal infec- } \\
\text { tions and tuberculosis } \\
{[44]}\end{array}$ & stem bark & $\begin{array}{l}\beta \text {-amyrin; alpinumisoflavone; genistein; laburnetin; } \\
\text { luteolin [44] }\end{array}$ & $\begin{array}{l}\text { (bark methanol extract) } \\
\text { M: } B c, B s t, C a, C g, E c l, M m \\
M, P m, S a[44]\end{array}$ \\
\hline & $\begin{array}{l}\text { Ficus cordata } \\
\text { Thunb. } \\
35446 / \text { HNC) }\end{array}$ & $\begin{array}{l}\text { filaris, diarrheal infec- } \\
\text { tions and tuberculosis } \\
{[44]}\end{array}$ & stem bark & $\begin{array}{l}\beta \text {-amyrin; } \beta \text {-sitosterol-3-O- } \beta \text {-o-glucopyranoside; cat- } \\
\text { echin; epiafzelechin [44] }\end{array}$ & $\begin{array}{l}\text { (bark methanol extract) } \\
\text { S: Ca, Cg, Ms, Mtb, Cf, Ec, Ecl, } \\
\text { Kp, Mm, Pm, Sd, St; M: Pa } \\
{[44]}\end{array}$ \\
\hline & $\begin{array}{l}\text { Ficus ovata } \\
\text { Vahl., } \\
\text { 26996SRF/ } \\
\text { Cam }\end{array}$ & $\begin{array}{l}\text { infectious diseases, gas- } \\
\text { trointestinal infections, } \\
\text { diarrhea, anti-poison } \\
\text { [109] }\end{array}$ & $\begin{array}{l}\text { leaves, } \\
\text { stem bark }\end{array}$ & $\begin{array}{l}\text { 3-friedelanone; taraxeryl acetate; betulinic acid; } \\
\text { oleanoï acid; 2'-hydroxyisoprunetin; 6,7-(2-isopro- } \\
\text { penyl furo)-5,2',4'-trihydroxyisoflavone; Cajanin; } \\
\text { protocatechuic acid [115] }\end{array}$ & $\begin{array}{l}\text { (Bark methanol extract) } \\
\text { M: } B c, C a, C f, E c, K p, P a, S a \\
\text { Sd, St [115] }\end{array}$ \\
\hline & $\begin{array}{l}\text { Morus mesozy- } \\
\text { gia Stapf. } \\
\text { (4228/SRFK) }\end{array}$ & $\begin{array}{l}\text { arthritis, rheumatism, } \\
\text { malnutrition, debility; } \\
\text { pain-killers, stomach } \\
\text { disorders, wound infec- } \\
\text { tions, gastroenteritis, } \\
\text { peptic ulcer, infectious } \\
\text { diseases }[78,116]\end{array}$ & stem bark & $\begin{array}{l}\text { marsformoxide B; moracin Q; moracin T; artocarpe- } \\
\text { sin; cycloartocarpesin; moracin R; moracin S; mora- } \\
\text { cin U; moracin C; moracin M [45] }\end{array}$ & $\begin{array}{l}\text { (bark methanol extract) } \\
\text { S: } B c, C a, E c ; \text { M: } K p, P a, S a \\
\text { Sd, Sf, St [45] }\end{array}$ \\
\hline & $\begin{array}{l}\text { Treculia acumi- } \\
\text { nata Baillon } \\
\text { (2921/SRF/ } \\
\text { Cam) }\end{array}$ & $\begin{array}{l}\text { treat skin diseases, den- } \\
\text { tal allergy, amoebic dys- } \\
\text { entery and AIDS [117, } \\
118]\end{array}$ & $\begin{array}{l}\text { leaves, } \\
\text { stem bark, } \\
\text { roots }\end{array}$ & $\begin{array}{l}\text { catechin; 6,9-dihydroxymegastigmane-3-one; 2,3- } \\
\text { dihydroxypropylhexadecanoate [62] }\end{array}$ & $\begin{array}{l}\text { (twigs methanol extract) } \\
\text { s: } B m, E a, E c l, S t ; \text { M: } C a, C g \\
C k, E c, P a, P m, P v, B s[62]\end{array}$ \\
\hline & $\begin{array}{l}\text { Treculia } \\
\text { africana Deca- } \\
\text { isne ( } 29053 / \\
\text { SRF/Cam) }\end{array}$ & $\begin{array}{l}\text { treat skin diseases, den- } \\
\text { tal allergy, amoebic dys- } \\
\text { entery and AIDS [117, } \\
118]\end{array}$ & $\begin{array}{l}\text { leaves, } \\
\text { stem bark, } \\
\text { roots }\end{array}$ & $\begin{array}{l}\text { phyllocoumarin; catechin; 6,9-dihydroxymegastig- } \\
\text { mane-3-one [62] }\end{array}$ & $\begin{array}{l}\text { (leaves methanol extract) } \\
\text { S: Bs, Ca, Cf, Ck, Ea, Ec, Kp, } \\
\mathrm{Mm}, P m, P v, S d, S f ; \mathbf{M}: B s t \\
C g, E a, P a, S a, S t[62]\end{array}$ \\
\hline & $\begin{array}{l}\text { Treculia } \\
\text { obovoidea } \\
\text { N.E. Brown } \\
(44055 / \text { HNC) }\end{array}$ & $\begin{array}{l}\text { treat skin diseases, den- } \\
\text { tal allergy, amoebic dys- } \\
\text { entery and AIDS [117, } \\
\text { 118] }\end{array}$ & $\begin{array}{l}\text { leaves, } \\
\text { stem bark, } \\
\text { roots }\end{array}$ & $\begin{array}{l}\text { psoralen; bergapten; 7-methoxycoumarin; 7-hy- } \\
\text { droxycoumarin; 4,2', 4'-trihydroxychalcone; 4,2',4'- } \\
\text { trihydroxy-3-prenylchalcone; 3-hydroxy-4-methoxy- } \\
\text { benzoic acid; O-[3-(2,2-dimethyl-3-oxo-2H-furan-5- } \\
\text { yl) butyl]bergaptol [22] }\end{array}$ & $\begin{array}{l}\text { (twigs methanol extract) } \\
\text { S: Bc, Bs, Ca, Cf, Ck, Pv; M: } \\
B m, B s t, C g, E c, E c l, K p, P a, S f \text {, } \\
\text { Sa, Sfl, St [22] }\end{array}$ \\
\hline $\begin{array}{l}\text { Hyperica- } \\
\text { ceae }\end{array}$ & $\begin{array}{l}\text { Harungana } \\
\text { madagas- } \\
\text { cariensis }^{\complement} \text { Lam. } \\
\text { ex. Poir (HNC } \\
\text { 32358) }\end{array}$ & - & leaves & $\begin{array}{l}\text { harunmadagascarin D, 1,7-dihydroxyxanthone } \\
\text { [119] }\end{array}$ & $\begin{array}{l}\text { crude extract not investi- } \\
\text { gated but harunmadagas- } \\
\text { carin } D \text { isolated from leaves } \\
\text { was active on } B \text {. cereus } \\
\text { [119] }\end{array}$ \\
\hline
\end{tabular}


Table 2 Plants used in Cameroon to treat infectious diseases, with evidence of their activities. (continued)

\begin{tabular}{|c|c|c|c|c|c|}
\hline Family & Species $^{a}$ & Traditional treatment & $\begin{array}{l}\text { Plant part } \\
\text { used }\end{array}$ & Bioactive (or potentially active) compounds & $\begin{array}{l}\text { Screened activity for } \\
\text { crude plant extract }\end{array}$ \\
\hline Meliaceae & $\begin{array}{l}\text { Turreanthus } \\
\text { manii (Baill.) } \\
(18312 / \text { SRF/ } \\
\text { Cam) }\end{array}$ & $\begin{array}{l}\text { infectious diseases } \\
{[120]}\end{array}$ & stem bark & $\begin{array}{l}\text { 16-acetoxy-12,15-epoxy-15 } \beta \text {-hydroxylabda-8 } \\
\text { (17),13-diene [120] }\end{array}$ & $\begin{array}{l}\text { (stem bark methanol ex- } \\
\text { tract) Q: Bs, Ec, Mmi, Cv, Ss } \\
{[120]}\end{array}$ \\
\hline \multirow[t]{3}{*}{$\begin{array}{l}\text { Meliantha- } \\
\text { ceae }\end{array}$} & $\begin{array}{l}\text { Bersama eng- } \\
\text { leriana Gurke } \\
(24725 / \mathrm{HNC})\end{array}$ & $\begin{array}{l}\text { cancer, spasms, infec- } \\
\text { tious diseases, male in- } \\
\text { fertility, diabetes [121] }\end{array}$ & $\begin{array}{l}\text { leaves, } \\
\text { stem bark, } \\
\text { roots }\end{array}$ & $\begin{array}{l}\text { not identified but flavonoids, phenols, triterpenes, } \\
\text { saponins and anthraquinones were detected in all } \\
\text { parts of the plant [122] }\end{array}$ & $\begin{array}{l}\text { (leaves methanol extract) } \\
\text { S: Bst, Ms, } K p, M m, P a, S t ; \\
\text { M: } B c, B s, C a, C f, C g, E c, E c l \\
\text { Sd, Sf, Sa, Sfl [122] }\end{array}$ \\
\hline & & & & & $\begin{array}{l}\text { (bark methanol extract) } \\
\text { S: Bs, Bst, } C a, C f, C g, E c, E c l, \\
K p, M m, M s, M t b, P a, S d, S f \text {, } \\
\text { Sa, Sfl, St [122] }\end{array}$ \\
\hline & & & & & $\begin{array}{l}\text { (roots methanol extract) } \\
\text { S: Bs, Bst, Ca, Cf, Cg, Ec, Ecl, } \\
\text { Kp, Mm, Ms, Mtb, Pa, Sd, Sf, } \\
\text { Sa, Sfl, St [122] }\end{array}$ \\
\hline \multirow[t]{4}{*}{ Ochnaceae } & $\begin{array}{l}\text { Campylosper- } \\
\text { mum glau- } \\
\text { cumc } \text { (Tiegh) } \\
\text { Farron } \\
\text { (28192/SRF/ } \\
\text { Cam) }\end{array}$ & - & stem bark & not identified & $\begin{array}{l}\text { (bark methanol extract) } \\
\text { W: Eh, Sa, Ssp [123] }\end{array}$ \\
\hline & $\begin{array}{l}\text { Ouratea sulca- } \\
\text { ta Van Tiegh } \\
\text { (ex Keay) } \\
(10133 / \text { SRF/ } \\
\text { Cam) }\end{array}$ & $\begin{array}{l}\text { upper respiratory tract } \\
\text { infections, dysentery, } \\
\text { diarrhea, toothache } \\
{[114]}\end{array}$ & leaves & $\begin{array}{l}\text { sulcatone A, 3-hydroxy-2,3-dihydroapigenyl-[l-4',O, } \\
\text { II-3']-dihydrokaempferol, amentoflavone [124] }\end{array}$ & $\begin{array}{l}\text { (leaves methanol extract) } \\
\text { W: } B s, S a, V a[124]\end{array}$ \\
\hline & & & & & $\begin{array}{l}\text { (leaves } \mathrm{CH}_{2} \mathrm{Cl}_{2}-\mathrm{MeOH} \text { ex- } \\
\text { tract) } \mathrm{M}: \mathrm{Bs} \text {, Sa, } \mathrm{Va}[124]\end{array}$ \\
\hline & $\begin{array}{l}\text { Ouratea turn- } \\
\text { area }(\text { Hook) } \\
\text { Hutch \& Dalz } \\
\text { (10134/SRF/ } \\
\text { Cam) }\end{array}$ & - & stem bark & not identified & $\begin{array}{l}\text { (bark methanol extract) } \\
\text { W: Eh, Sa, Ssp [123] }\end{array}$ \\
\hline $\begin{array}{l}\text { Poaceae/ } \\
\text { Gramineae }\end{array}$ & $\begin{array}{l}\text { Cymbopogon } \\
\text { citratus(DC) } \\
\text { Stapf. (18628/ } \\
\text { SRF/Cam) }\end{array}$ & fungal infections [109] & leaves & essential oil, with non-identified components [109] & (essential oil) Q: Ao [109] \\
\hline $\begin{array}{l}\text { Rhamna- } \\
\text { ceae }\end{array}$ & $\begin{array}{l}\text { Maesopsis emi- } \\
\text { nii (Engler) } \\
\text { (234/SRF/ } \\
\text { Cam) }\end{array}$ & $\begin{array}{l}\text { diuretic, purgative, em- } \\
\text { etic, and antidiarrhetic, } \\
\text { abortifacient }[125,126]\end{array}$ & stem bark & $1 \alpha, 3 \beta$-dihydroxybauer-7-en-28-oic acid [125] & $\begin{array}{l}\text { crude extract was not in- } \\
\text { vestigated; a diterpenoid } \\
1 \alpha, 3 \beta \text {-dihydroxybauer-7- } \\
\text { en-28-oic acid isolated from } \\
\text { the stem bark was active on } \\
\text { B. cereus [125] }\end{array}$ \\
\hline \multirow[t]{4}{*}{ Rutaceae } & $\begin{array}{l}\text { Tecla afzelii } \\
\text { Engl. (10674/ } \\
\text { SRF/Cam) }\end{array}$ & $\begin{array}{l}\text { wound infections, ab- } \\
\text { dominal pains, cough, } \\
\text { fever, asthma [127] }\end{array}$ & stem bark & kokusaginine; maculine; kolbisine; lupeol [41] & $\begin{array}{l}\text { (bark methanol extract) } \\
\text { S: } B s, C a, C g, E c, M a, S t ; \\
\text { M: } M s[41]\end{array}$ \\
\hline & $\begin{array}{l}\text { Oriciopsis gla- } \\
\text { berrima Engl. } \\
(1888 / \text { HNC) }\end{array}$ & $\begin{array}{l}\text { infections, hypotension, } \\
\text { mycoses, dermatitis } \\
{[114]}\end{array}$ & Stem bark & oriciacridone A and B, lichexanthone [128] & $\begin{array}{l}\text { (bark } \mathrm{CH}_{2} \mathrm{Cl}_{2}-\mathrm{MeOH} \text { ex- } \\
\text { tract) Q: } B s, \mathrm{Ca}, \mathrm{Cv}, \mathrm{Cs}, \mathrm{Mmi} \text {, } \\
\text { Sa, Ss, Sv [128] }\end{array}$ \\
\hline & $\begin{array}{l}\text { Zanthoxylum } \\
\text { leprieurii }\end{array}$ & $\begin{array}{l}\text { gonorrhea, kidney pain, } \\
\text { sterility [77] }\end{array}$ & $\begin{array}{l}\text { not speci- } \\
\text { fied }\end{array}$ & $\begin{array}{l}\text { Not identified but essential oils from fruits con- } \\
\text { tained trans- } \alpha \text {-ocimene; } \alpha \text {-terpinolene; } 3 \text { - } \delta \text {-carene; } \\
\text { limonene; myrcene; } \alpha \text {-pinene; } p \text {-cymene [77] }\end{array}$ & $\begin{array}{l}\text { (fruits essential oils) S: Sa } \\
\text { [77] }\end{array}$ \\
\hline & $\begin{array}{l}\text { Zanthaxylum } \\
\text { xanthoxyloides }\end{array}$ & $\begin{array}{l}\text { enteritis, dysentery, } \\
\text { diarrhea, guinea worm, } \\
\text { uretritis and as an anti- } \\
\text { odontalgic [77] }\end{array}$ & $\begin{array}{l}\text { not speci- } \\
\text { fied }\end{array}$ & $\begin{array}{l}\text { not identified but essential oils from fruits contained } \\
\alpha \text {-pinene; trans- } \beta \text {-ocimene; citronellol; sabinene; } \\
\text { myrcene; limonene; cytronellyl acetate; } \alpha \text {-phellan- } \\
\text { drene [77] }\end{array}$ & $\begin{array}{l}\text { (fruits essential oils) S: } E c \text {, } \\
B c, B s, A f, K p, S a, S f[77]\end{array}$ \\
\hline $\begin{array}{l}\text { Sapota- } \\
\text { ceae }\end{array}$ & $\begin{array}{l}\text { Tridesmoste- } \\
\text { mon omphalo- } \\
\text { carpoides Engl. } \\
(3829 / \mathrm{HNC})\end{array}$ & $\begin{array}{l}\text { gastroenteritis, skin } \\
\text { lesions [129] }\end{array}$ & stem bark & $\begin{array}{l}\text { not identified but preliminary phytochemical stud- } \\
\text { ies reported the presence of alkaloids, phenols, } \\
\text { polyphenols, saponins, tannins, triterpenes, anthra- } \\
\text { quinones and steroids in bark methanolic extract } \\
\text { and their variation in active fractions [129] }\end{array}$ & $\begin{array}{l}\text { (bark methanol extract) } \\
\text { S: } E c ; \text { M: Ca, Ck, Sd, Kp, Sa, Sf } \\
{[129]}\end{array}$ \\
\hline
\end{tabular}


Table 2 Plants used in Cameroon to treat infectious diseases, with evidence of their activities. (continued)

\begin{tabular}{|c|c|c|c|c|c|}
\hline Family & Species $^{a}$ & Traditional treatment & $\begin{array}{l}\text { Plant part } \\
\text { used }\end{array}$ & Bioactive (or potentially active) compounds & $\begin{array}{l}\text { Screened activityb for } \\
\text { crude plant extract }\end{array}$ \\
\hline $\begin{array}{l}\text { Solana- } \\
\text { ceae }\end{array}$ & $\begin{array}{l}\text { Slonanum } \\
\text { tovum Sw. } \\
(49427 / \mathrm{HNC})\end{array}$ & $\begin{array}{l}\text { bacterial and fungal in- } \\
\text { fections, HIV, herpes } \\
\text { simplex virus type I and } \\
\text { II infections [76, 130- } \\
\text { 132] }\end{array}$ & $\begin{array}{l}\text { leaves, } \\
\text { stem bark, } \\
\text { fruits }\end{array}$ & $\begin{array}{l}\text { steroidiques glycosides, chlorogenone, neochloro- } \\
\text { genone [131, 132], solasodine, lupeol [76] }\end{array}$ & $\begin{array}{l}\text { (fruits ethanol } 70 \% \text { extract) } \\
\text { M: Ca, Ck, Ec, Ng, Pa, Pv, Sd, } \\
\text { Sfl, Sa, St, Sf [76] }\end{array}$ \\
\hline $\begin{array}{l}\text { Zingibera- } \\
\text { ceae }\end{array}$ & $\begin{array}{l}\text { Zingiber } \\
\text { officinale }\end{array}$ & $\begin{array}{l}\text { infectious diseases, res- } \\
\text { piratory tract infections } \\
\text { [98] }\end{array}$ & roots & not yet identified & $\begin{array}{l}\text { (roots ethanol extract) S: } \\
\text { Sa, Sp, Spn, Hi [98] }\end{array}$ \\
\hline
\end{tabular}

a HNC or SRFK: Cameroon national herbarium code; b Screened activity: significant, S: MIC < $100 \mu \mathrm{g} / \mathrm{mL})$, moderate $(\mathrm{MIC}: 100<\mathrm{MIC} \leq 625 \mu \mathrm{g} / \mathrm{mL}), \mathrm{weak}(\mathrm{W}$ : MIC >625 $\mu \mathrm{g} / \mathrm{mL}), \mathrm{Q}$ : qualitative activity based on inhibition zone determination; An: Aspergillus niger; Af: Aspergillus flavus; Asp: Alternaria sp.; Ao: Aspergillus ochraceus; Bc: Bacillus cereus; Bm:Bacillus megaterium; Bs: Bacillus subtilis; Bst: Bacillus stearothermophilus; Ca: Candida albicans; Cn: Cryptococcus neoformans; Cf: Citrobacter freundii; Cg: Candida glabrata; Cgl: Corynebacterium glutamicum; Ck: Candida krusei; Cs: Chlorella sorokiniana; Csp; Cladosporium sp.; Ct: Candida tropicalis; Cv: Chlorella vulgaris; Ea: Enterobacter aerogenes; Ec: Escherichia coli; ECl: Enterobacter cloacae; Eh: Enterococcus hirae; Fsp: Fusarium sp; Gc: Geotrichum candidum; Hi: Haemophilus influenzae; Kp: Klebsiella pneumoniae; Ma: Microsporum audouinii; Mm: Morganella morganii; Mmi: Mucor miehei; Ms: Mycobacterium smegmatis; Mtb: Mycobacterium tuberculosis; Ng: Neisseria gonorrhoeae; Pa: Pseudomonas aeruginosa; Pm: Proteus mirabilis; Pv: Proteus vulgaris; Psp: Penicillium; Pv: Penicillium verrucosum; Sa: Staphylococcus aureus; Sd: Shigella dysenteriae; Sf: Streptococcus faecalis; Sm: Streptococcus mutans; San: Streptococcus anginosus; So: Streptococcus oralis; Spn: Streptococcus pneumoniae; Sp: Streptococcus pyogenes; Sfl: Shigella flexneri; Sp: Streptococcus pneumoniae; Ss: Scenedesmus subspicatus; St: Salmonella typh; Sv: Streptomyces viridochromogenes; Tm: Trichophyton mentagophytes; Tr: Trichophyton rubrum; Va: Vibrio anguillarium; SSp: Staphylococcus saprophyticus; c Plant with no reference for the use in the treatment of infectious diseases, but that extract or derived product showed antimicrobial activity

diversity of classes, such as alkaloids, terpenoids, peptides and phenolics [18]. Numerous assay systems and organisms have been used to screen plant extracts and constituents of active plants for antimicrobial activity. The microbroth dilution method seems to be more appropriate when investigating the activity of compounds. However, this method has several advantages compared to another method used in the past; the agar diffusion method. The microbroth dilution method is quantitative, allows the use of small quantities of compounds or plant extracts as well as culture media, and is well adapted for drugs intended for systemic use [19]. Colorimetric microbroth techniques using various reagents such as tetrazolium salts [20,21], or color indicators [22] allow easy MIC detection and increase the credibility of this method. For the antimycobacterial tests of plant-derived substances, a number of bioassay systems has been used including agar diffusion and dilution assays, radiorespirometry (using the BACTEC 460 instrument), and broth macro- and micro-dilution assays to reporter gene assays [14].

\section{Biological Activity Screening of Plant Extracts for Antimicrobial Effects in Cameroon \\ $\nabla$}

Plants extracts are widely used in many parts of Cameroon to treat infectious diseases or related symptoms including abdominal pains, itching, urinary and respiratory ailments, fever and coughing, diarrhea. Adjanohoun et al. [23] provided a useful review of the traditional use of medicinal plants in Cameroon, although much work remains to be done regarding the documentation of existing ethnobotanical knowledge. Cameroon possesses a very rich and diverse flora, with an estimated 8260 species [24]. This paper is the first review on Cameroonian medicinal plants and derived products as a source of antimicrobial agents. It is important to note that a minimal inhibitory concentration (MIC) value of $100 \mu \mathrm{g} / \mathrm{mL}$ was used as a criterion for antimicrobial activity classification in accordance with some authors who consider a MIC value between $100-200 \mu \mathrm{g} / \mathrm{mL}$ as positive for plant extracts [25-29]. The plants with scientific reports on their activities of any part or derived products against microorganisms
( Table 1 ) are summarized in $\bullet$ Table 2 . In this review, the activity of plant extracts or compounds will also be discussed, but not classified if the documented results were based only on the inhibition zone determinations. However, in this paper, the activity of plant extracts will be classified as significant (MIC $<100 \mu \mathrm{g} /$ $\mathrm{mL})$, moderate $(100<\mathrm{MIC} \leq 625 \mu \mathrm{g} / \mathrm{mL}$ ) or weak (MIC $>625 \mu \mathrm{g}$ / $\mathrm{mL})$.

It appears from the results of $\bullet$ Table 2 that a number of crude extracts were significantly active. Some of them include extracts of Bersama engleriana, Dorstenia angusticornis, Dorstenia turbinata, Dorstenia barteri, Newbouldia laevis, Vismia laurentii, Vismia guineensis, etc. Numerous active metabolites were isolated from these plants and include several classes.

\section{Antimicrobial Compounds from Cameroonian Medicinal Plants}

Most of the antimicrobial substances isolated from Cameroonian medicinal plants belong to three main classes of secondary metabolites, i.e., terpenoids, phenolic compounds and alkaloids. The classification criterion is highly stringent, but several authors agree to keep the level of $10 \mu \mathrm{g} / \mathrm{mL}$ or $50 \mu \mathrm{M}$ as the threshold for acceptable activity $[30,31]$. In this study, we will set the value as follows: significant activity (MIC $<10 \mu \mathrm{g} / \mathrm{mL}$ ), moderate $(10<$ MIC $\leq 100 \mu \mathrm{g} / \mathrm{mL}$ ), and low or negligible (MIC $>100 \mu \mathrm{g} / \mathrm{mL}$ ).

\section{Terpenoids}

Terpenoids are the largest and most widespread class of secondary metabolites, mainly in plants and lower invertebrates. A few of them have been used for therapeutic purposes for centuries; but in recent decades the level of research activity in isolating and studying new terpenoids has shown no sign of abating [32]. Generally, terpenoids have low antimicrobial potentials, compared to phenolic compounds. Several terpenoids have been isolated and tested, but a few of them presented an acceptable activity, both antibacterial and antifungal. Nevertheless, some of the terpenoids such as the triterpenoid betulinic acid has been shown to inhibit HIV [33]. Two terpenoids, cymbopogonol and 
citral showed antifungal activity against $C$. albicans [34]. Also the diterpenoid trichorabdal A [35] was found to be active against Helicobacter pylori. Plant oils, which contain terpenoids, have shown increasing promise in vivo, inhibiting multiple species of bacteria. For example, cinnamon oil has shown broad-spectrum activity against Pseudomonas aeruginosa [36]. Also, John et al. [37] found that plant oils from Neolitsea foliosa, which also exhibited some antibacterial properties, included sesquiterpenes such as $\beta$-caryophyllene. A terpenoid, 3-oxo-(20S,24S)-epoxydammarane 19,25-diacetate isolated from the barks of Caesalpinia pulcherrina also exhibited significant antibacterial activity and a prominent antifungal activity [38]. The mechanism of action of terpenoids is not fully understood but is speculated to involve membrane disruption by the lipophilic compounds. Among the terpenoids isolated from Cameroonian medicinal plants, both hardwiickic acid (1) and friedelin (2) ( Fig. 1) exhibited interesting antimicrobial effects on gram-positive bacteria and against the gram-negative bacteria $[39,40]$. Compound 1 however, presented moderate activity on many other bacterial species and Candida spp. [39]. Compound 2 also presented a significant antibacterial activity against $C$. freundii, M. morganii, Shigella spp., Proteus spp., P. aeruginosa, Bacillus spp., S. faecalis and Candida spp. [40]. Lupeol and many others triterpenoids were also isolated from Cameroonian plants and tested on a panel of bacteria and yeasts, but most of them exhibited poor activities [41].

\section{Phenolic compounds}

Flavonoids: Several flavonoids isolated from Cameroonian medicinal plants have been reported for their antimicrobial activities ( Fig. 2). Such compounds comprise largely chalcones, flavones and isoflavones. Chalcones were isolated primarily from plants of the family Moraceae and the genus Dortenia such as Dorstenia angusticornis [42], Dorstenia elliptica [43], Dorstenia turbinata [6], and Dorstenia barteri [5]. Among the chalcones, diprenylated compounds such as angusticornin B (3) and bartericin A (4) were reported to be very active vis-à-vis many gram-positive and gram-negative bacteria as well as yeasts such as C. albicans, C. glabrata and C. krusei [42]. It has been demonstrated that hydroxylation of the prenyl groups of stipulin (5) leads to compounds 3 and 4, inducing a significant increase of the antimicrobial activity [42]. Mbaveng et al. [5] also demonstrated that transposition of prenyl from the 5 '- (stipulin) to the 3 '-position leads to kanzanol C (6), and induces an increase of antimicrobial activity, with compound 6 exhibiting significant antimicrobial activities against M. morganii and S. flexneri while $\mathbf{5}$ was not so active. A monoprenylated chalcone, isobavachalcone (7), was more active than most of the diprenylated chalcones tested so far, with significant inhibitory effects observed on several bacteria and fungi [5]. Cyclization of this molecule, leading to 4-hydroxylonchocarpin (8), induced a significant reduction of the activity [5]. Kuete et al. [22] also demonstrated that the shift of the prenyl group from C-3 of compound 7 to position 3' (4,2',4'-trihydroxy3-prenylchacone; 9), reduced the specificity of compound 9 against gram-negative bacteria, while activity remained significant on the gram-positive bacteria and yeasts. Also, the absence of prenyl groups leading to 4,2',4'-trihydroxychacone (10) further reduced this activity. This allows us to conclude that the prenyl group plays an important role in the activity and selectivity of microorganisms to chalcones. Some flavones such as gancaonin Q (11) and kaempferol (12) were significantly active against E. aerogenes, S. dysenteriae and Bacillus spp. [40,42]. Several other flavonoids have shown moderate antimicrobial activities. This in- cludes luteolin, catechin, epiafzelcetin, phyllocoumarin, amentoflavone, artocarpesin, and cycloartocarpesin [5,22,42,44,45]. Many bioactive isoflavonoids were also isolated from Cameroonian medicinal plants. Although isoflavonoids such as laburnetin (13) [44] showed significant activity against $M$. tuberculosis, activities against gram-positive and gram-negative bacteria and fungi, and those of genistein, alpium isoflavone, 2'-hydroxyisoprunetin, 6,7-(2-isopropenylfuro)-5,2',4'-trihydroxyisoflavone and cajanin were found to be selective, moderate or negligible $[44,45]$. Similarly to chalcones, it has also been demonstrated that the cyclization of flavones (e.g., artocarpesin to cycloartocarpesin) reduced the antimicrobial activity [45].

Arylbenzofuran: Arylbenzofurans ( Fig. 1) were isolated from Morus mesozygia, including 2-arylbenzofurans of the moracin series (C, M, Q R, S, T and U) $[45,46]$. Although very few arylbenzofurans have so far been isolated, it has been shown that compounds of the moracin series have moderate activities. Nevertheless, some of them such as moracin T (14) were very active (MIC $<10 \mu \mathrm{g} / \mathrm{mL}$ ) on E. coli, S. dysenteriae, P. aeruginosa, K. pneumoniae, S. typhi, B. cereus, S. aureus, S. faecalis, and C. albicans [45]. Significant activities of moracin $\mathrm{M}(\mathbf{1 5})$ against $P$. aeruginosa, moracin $U$ (16) against $E$. coli, and B. cereus and moracin C (17) against $S$. dysenteriae, P. aeruginosa and $S$. typhi were also reported [45]. The antimicrobial activities of other 2-arylbenzofurans such as 6,6'-dihydroxy-4'-methoxy-2-arylbenzofuran, cicerfuran and benzofuran derivatives [46] have, however, been documented [47]. Kuete et al. [45] demonstrated that the prenylation of arylbenzofuran increases the antimicrobial activity, with monoprenylated compounds being generally more active. Similarly to chalcones and flavones, it was shown that the degree of activity depends on the position of the prenyl group, with compound 14 (with C-4 prenylation) being more active than compound $\mathbf{1 7}$ (with C-4' prenylation) [45]. It was also reported that the cyclization of arylbenzofurans reduces their antimicrobial activities [45].

Quinones: Several naphthoquinones isolated from Cameroonian medicinal plants were reported for their activities against bacteria and fungi ( $\odot$ Fig. 3). MICs $<10 \mu \mathrm{g} / \mathrm{mL}$ were documented for many of them including lapachol (18), 2-acetylfuro-1,4-naphthoquinone (19), 2-hydroxy-3-methoxy-9,10-dioxo-9,10-dihydroanthracene-1-carbaldehyde (20), newbouldiaquinone (21) [48]. Very interesting activities of plumbagin (22), diospyrone (23) and crassiflorone (24) were reported against $M$. tuberculosis, M. smegmatis and N. gonorrhoeae [49]. Several other quinones (๑ Fig. 3) also demonstrated significant antifungal and antibacterial activities, namely newbouldiaquinone A (25), vismiaquinone $C$ (26), vismiaquinone (27), 3-geranyloxy-6-methyl-1,8dihydroxyanthraquinone (28), 1,8-dihydroxy-6-methoxy-3methylanthraquinone (29), and bivismiaquinone (30) [40,48]. Despite the important structural differences between these quinones, the antibacterial and antifungal activities were found to be significant and close to each other, indicating that the presence of the skeleton of naphthoquinones and anthraquinones is the basis of their antimicrobial activities. It has been demonstrated that quinones complex irreversibly with nucleophilic amino acids of microbial proteins, leading to the loss of function and consequently to the death of the pathogens [50]. The reactivity of cluster-based quinones explains why most of these molecules exert significant antimicrobial activities. However, previous studies [48] also proved that the cyclization and the prenylation of naphthoquinones act on the specificity of the antimicrobial activity. 


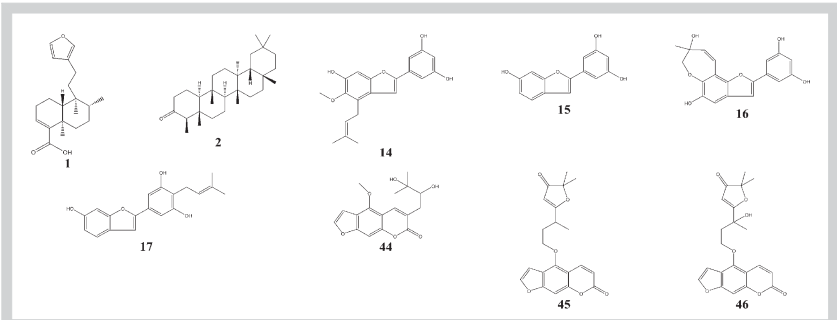

Fig. 1 Antimicrobial terpenoids (1 and 2), arylbenzofurans (14 - 17) and coumarins (44-46).

Xanthones: Several xanthones ( Fig. 4) with good antimicrobial properties have been isolated from various medicinal plants of Cameroon. They have been isolated mostly from plants of the family Guttiferae, including members of the genus Garcinia such as Garcinia smeathmanii [51] and Garcinia polyantha [52], and the genus Vismia such as Vismia laurentii [40], and Vismia guineensis [53]. Many of them, such as cheffouxanthone (31) smeathxanthone B (32) [51], 6-deoxyisojacareubin (33), $O^{1}$-demethyl-3',4'-deoxypsorospermin-3,4'-diol (34), 1,3,7-trihydroxyxanthone (35) [40], laurentixanthone A (36), and laurentixanthone B (37) [54] presented selective and significant activities on several bacteria and yeasts of the genus Candida. Banganxanthone A (38) presented a significant antimycobacterial activity against $M$. tuberculosis and $M$. smegmatis [52]. Azebaze et al. [55] reported allaxanthone D (39) as a significantly active antimicrobial xanthone. Other bioactive compounds of this class were also documented. These include 1,3,6,7-tetrahydroxy-2-(3methylbut-2-enyl)xanthone (40) that was active against E. cloacae, K. pneumoniae, P. aeruginosa, S. faecalis, S. aureus, B. megaterium, B. subtilis and C. glabrata [55]. Compounds such as globulixanthones C (41), D (42) and E (43) also exhibited antimicrobial activities against S. aureus, B. subtilis and Vibrio anguillarium [56].

Coumarins: Several coumarins have antimicrobial properties [57-61]. They have been found to stimulate macrophages [59], which could have an indirect negative effect on infections. More specifically, coumarin has been used to prevent recurrences of cold sores caused by HSV-1 in humans [57]. Phytoalexins, which are hydroxylated derivatives of coumarins, are produced in carrots in response to fungal infection and can be presumed to have antifungal activity [58]. Osthenol also exhibited good activity against gram-positive bacteria [60]. Most of the coumarins isolated so far from Cameroonian medicinal plants ( $\bullet$ Fig. 1 ) were found in plants of the genus Treculia (Moraceae), including Treculia africana, Treculia acuminata and Treculia obovoidea [22,62]. They exhibited moderate antibacterial and antifungal activities $[6,22]$. Nevertheless, compounds such as 5-methoxy-3-(3-methyl-2,3-dihydroxybutyl)psoralen (44), 5-methoxy-3-[3-( $\beta$-glucopyranosyloxy)-2-hydroxy-3-methylbutyl]psoralen (45) exhibited significant antifungal activities with MIC values comparable to those of nystatin [6]. O-[3-(2,2-Dimethyl-3-oxo-2H-furan-5yl)butyl]bergaptol (46) also had very good, but selective antimicrobial activities against yeasts of the genus Candida, gram-postive and gram-negative bacteria [22].

Other phenols, benzophenones, ellagic acid derivatives: Several other compounds including simple phenolics, benzophenones, cinnamic and ellagic acid derivatives ( $\bullet$ Fig. 5) were identified as active antimicrobial principles of some Cameroonian medicinal plants. Though simple phenolics such as 4-hydroxy-3-me-

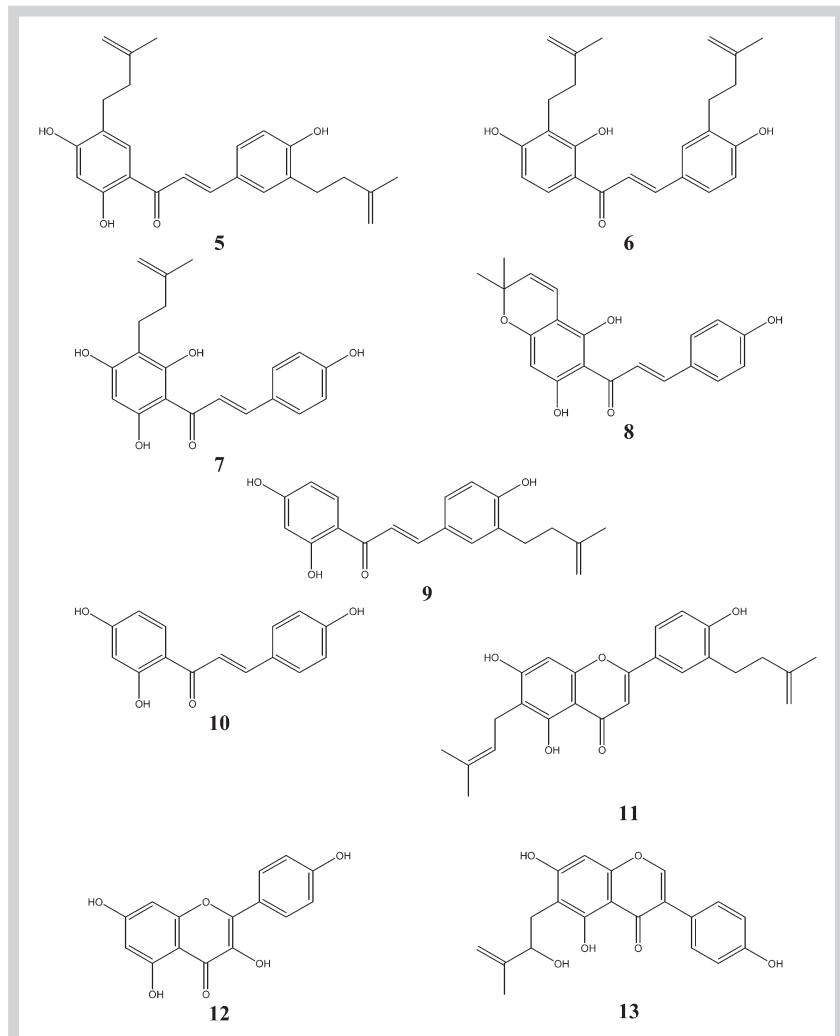

Fig. 2 Antimicrobial flavonoids (3 - 13).

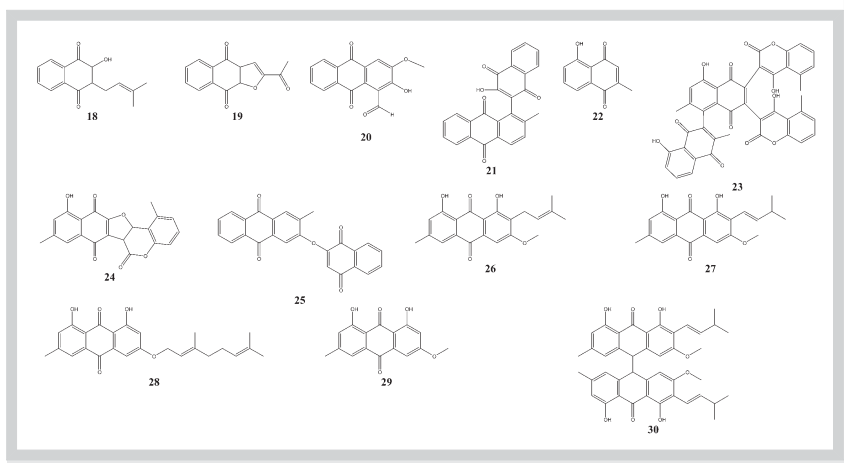

Fig. 3 Antimicrobial quinones $(\mathbf{1 8} \mathbf{- 3 0})$.

thoxybenzaldehyde, 4-methoxyphenol and 3-hydroxy-4-methoxybenzoic acid had weak inhibitory potentials [22], benzophenones presented better activities [51]. This is the case of guttiferone I (47) with MIC $<10 \mu \mathrm{g} / \mathrm{mL}$ reported on C. freundii, E. cloacae, P. vulgaris, B. megaterium and $S$. faecalis [51]. Isoxanthochymol (48) also exhibited significant activity against $B$. cereus and $B$. stearothermophilus [51]. Ellagic acid (49) and its derivatives 3,4di-O-methylellagic acid (50) and 3,3',4'-tri-O-methylellagic acid (51) were significantly active against a wide range of bacteria and yeasts [39].

\section{Alkaloids}

Natural alkaloids are known for their anti-infective activities. A review of anti-HIV compounds of plant origin by Cos et al. [63] summarized published data on several classes of alkaloids including naphthylisoquinoline alkaloid dimers (michellamines A- 


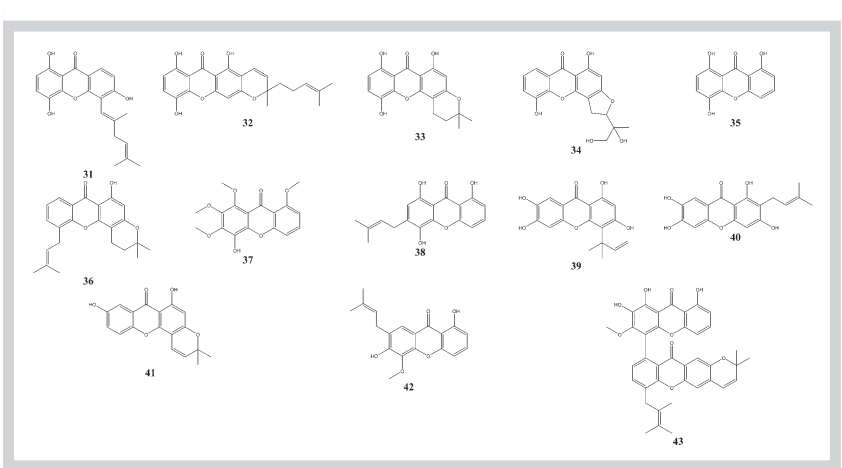

Fig. 4 Antimicrobial xanthones (31 - 43).

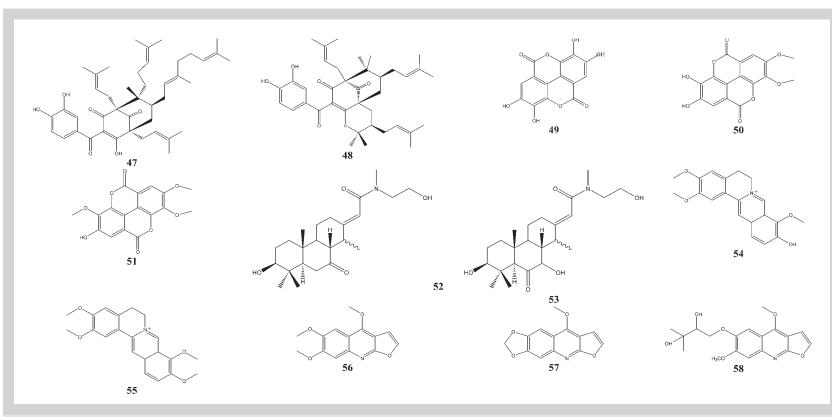

Fig. 5 Antimicrobial benzophenones $(47,48)$, ellagic acid $(49)$ and its derivatives $(\mathbf{5 0}, \mathbf{5 1})$, and alkaloids $(\mathbf{5 2}-\mathbf{5 8})$.

F [64,65], nitrogen-containing sugar analogues (castanospermine and 1-deoxynojirimycin) [66,67], sesquiterpene pyridine alkaloids (triptonines A and B) [68], the $\beta$-carboline alkaloid harman [69], and the carbazole alkaloid, siamenol [70]. Diterpene alkaloids, commonly isolated from the plants of the Ranunculaceae, or buttercup [71] family [72], were found to have antimicrobial properties. Berberine, an important representative of the alkaloid group was also found to be active against $S$. aureus with RNA being suggested as its possible target [73]. Compared with phenolics and terpenoids, very few antimicrobial alkaloids have been isolated so far from Cameroonian medicinal plants. This is due to the fact that few numbers of the plant families contain this class of compounds [74]. Alkaloids from Cameroonian medicinal plants ( Fig. 5) were mostly isolated in three families including Rutaceae (Tecla afzelii) [34], Caesalpiniaceae (Erythrophleum suaveolens) [75] and Apocynaceae (Tabernaemontana crassa) [76]. The presence of alkaloids in these plant families has also been reported [74]. Amongst the antimicrobial alkaloids ( Fig. 5) isolated from such plants, norcassaide (52) and norerythrosuaveolide (53) isolated from Erythrophleum suaveolens exerted significant inhibitory (MIC $<10 \mu \mathrm{g} / \mathrm{mL}$ ) activities against selected microbial strains like K. pneumoniae, $N$. gonorrhoeae, C. albicans, and C. krusei [75]. Dehydrocorydalmine (54) and palmatine (55) from Tabernaemontana crassa also presented a good activity on $N$. gonorrhoeae and C. krusei [76]. Kokusaginine (56), maculine (57) and nkolbisine (58) isolated from the stem bark of Tecla afzelii presented rather low or moderate activities, but MICs below $10 \mu \mathrm{g} / \mathrm{mL}$ were recorded on some bacterial species [41].

\section{Conclusions}

$\nabla$

This review, the first of its kind on Cameroonian medicinal plants as potential antimicrobials, is intended to serve as the scientific baseline information for the use of the documented plants, as well as a starting point for future studies, leading to the production of improved plant medicines. The paper also draws attention to some active metabolites, which could probably lead to new antimicrobial drugs. The present review will inevitably show the richness of Cameroon medicinal flora as antimicrobial resources and demonstrates that many of them that are used traditionally are effective. Some of the Cameroonian plant extracts distinguished themselves by their exceptional inhibitory power on both bacteria and fungi. Among these are Bersama engleriana, Dorstenia angusticornis, Dorstenia barteri, Diospyros canaliculata, Diospyros crassiflora, Newbouldia laevis, and Ficus cordata. Some of the isolated compounds were also highly active. This was the case for isobavachalcone, kanzanol $\mathrm{C}$ and 4-hydroxylonchocarpin isolated from Dorstenia spp., plumbagin, crassiflorone and diospyrone isolated from Diospyros spp., and also newboudiaquinone, lapachol and newbouldiaquinone isolated from Newbouldia laevis. Some of the bioactive compounds such as diospyrone (23), crassiflorone (24), newboudiaquinone (21), newbouldiaquinone A (25), laurentixanthone A (36), laurentixanthone B (37), norcassaïde (49), norerythrosuaveolide (50) [50], smeathxanthone B (32), cheffouxanthone (31) banganxanthone A (38), moracin $T$ (14), moracin $U$ (16), globulixanthones C (41), D (42) and E (43) and many other compounds were isolated and characterized for the first time in Cameroonian medicinal plants. Presently, there is an urgent necessity for standardizing plant drugs from the investigated plants, as their use is still empirical. There is also an urgent requirement to standardize methods and cut-off points for describing antimicrobial activities, as some authors report activities of extracts at more than $10 \mathrm{mg} / \mathrm{mL}$ while others, including ourselves, believe that only MIC values less than $100 \mu \mathrm{g} / \mathrm{mL}$ (for extracts) and $10 \mu \mathrm{g} / \mathrm{mL}$ (for compounds) are worthy of the label active. Other recommendations are to include a parallel screening of mammalian cytotoxicity tests to preclude nonspecific cytotoxicity from being interpreted as antimicrobial efficacy following in vitro screening. This is being done in some studies to provide useful selective data, but few research teams in the country are concerned. The study of the mechanism of action and resistance was initiated in our research team at the University of Dschang on active metabolites or extracts, and we recommend that where antimicrobial testings are going on, this should be a priority.

\section{Acknowledgements \\ $\nabla$}

VK is grateful to Drs. H.M. Poumale Poumale, J. Komguem, R. N. Manfouo, J. Gangoué Pieboji, J. G. Tangmouo, A.T. Mbaveng; (Faculty of Science, University of Yaoundé I) and P. Lunga (University of Dschang) for their support and advice.

\section{References}

1 Céspedes CL, Avila G, Martinez A, Serrato B, Calderon-Mugica JC, Salgado-Garciglia R. Antifungal and antibacterial activities of Mexican tarragon (Tagetes lucida). J Agric Food Chem 2006; 54: 3521-3527

2 Penso G. Index Plantarum Medicinalium Totius Mundicorunque Synoni Morum. Milano: Organizacione Editoriale Medico Farmaceutica; 1982 
3 Biyiti L, Pesando D, Puiseux-Dao S. Antimicrobial activity of two flavanones isolated from the Cameroonian plant Erythrina sigmoidea. Planta Med 1988; 54: 126-128

4 Kuete V, Tangmouo JG, Beng VP, Nguemfo EL, Mofo F, Etoa FX, Lontsi D, Samreen IA. Activités antibactérienne et cytotoxique in vitro de différents extraits des écorces du tronc de Diospyros canaliculata (Ebenaceae). West Afr J Pharmacol Drug Res 2004; 30: 22-25

5 Mbaveng AT, Ngameni B, Kuete V, Konga Simo I, Ambassa P, Roy R, Bezabih M, Etoa FX, Ngadjui BT, Abegaz BM, Meyer JJM, Lall N, Beng VP. Antimicrobial activity of the crude extracts and five flavonoids from the twigs of Dorstenia barteri (Moraceae). J Ethnopharmacol 2008; 116: 483-489

6 Ngameni B, Kuete V, Konga Simo I, Mbaveng AT, Awoussong PK, Patnam $R$, Roy R, Ngadjui BT. Antibacterial and antifungal activities of the crude extract and compounds from Dorstenia turbinata (Moraceae). S Afr J Bot 2009; 75: 256-261

7 WHO. WHO Guideline for the Assessment of herbal Medicines, WHO expert committee on specification for pharmaceutical preparation. Technical Report series No 863. Geneva: WHO; 1996

8 GLOBAL: Microbes don't know geography - WHO report. http://www. irinnews.org/Report.aspx?Reportld=73901. Accessed on 2 August 2009

9 WHO. International spread of disease threatens public health security: The world health report 2007 focuses on building a safer future. Geneva. Available at: (http://www.who.int/mediacentre/news/releases/ 2007/pr44/en/) 2007. Accessed on August 02, 2009

10 Zager EM, McNerney R. Multidrug-resistant tuberculosis. BMC Infect Dis 2008; 8: 10

11 Cameroon major infectious diseases. http://www.indexmundi.com/ cameroon/major_infectious_diseases.html. Accessed on August 02, 2009

12 Ammah A, Nkuo-Akenji T, Ndip R, Deas JE. An update on concurrent malaria and typhoid fever in Cameroon. Trans R Soc Trop Med Hyg 1999; 93: $127-129$

13 Noeske J, Kuaban C, Cunin P. Are smear-positive pulmonary tuberculosis patients a 'sentinel' population for the HIV epidemic in Cameroon? Int J Tuberc Lung Dis 2004; 8: 346-351

14 McGaw LJ, Lall N, Meyer JJM, Eloff JN. The potential of South African plants against Mycobacterium infections. J Ethnopharmacol 2008; 119: $482-500$

15 Jones KDJ, Hesketh T, Yudkin J. Extensively drug-resistant tuberculosis in sub-Saharan Africa: an emerging public-health concern. Trans R Soc Trop Med Hyg 2008; 102: 219-224

16 Donald PR, Sirgel FA, Venter A, Parkin DP, Seifart HI, van deWal BW, Maritz JS, Fourie PB. Early bactericidal activity of antituberculosis agents. Expert Rev Anti Infect Ther 2003; 1: 141-145

17 Cragg GM, Newman DJ, Snader KM. Natural products in drug discovery and development. J Nat Prod 1997; 60: 52-60

18 Cowan MM. Plant products as antimicrobial agents. Clin Microbiol Rev 1999; $12:$ 564-582

19 Chung GAC, Aktar Z, Jackson S, Duncan K. High-throughput screen for detecting antimycobacterial agents. Antimicrob Agents Chemother 1995; 39: 2235-2238

20 Eloff JN. A sensitive and quick microplate method to determine the minimal inhibitory concentration of plant extracts for bacteria. Planta Med 1998; 64: 711-713

21 Babula P, Adam V, Kizek R, Sladky Z, Havel L. Naphthoquinones as allelochemical triggers of programmed cell death. Environ Exp Bot 2009; 65: 330-337

22 Kuete V, Metuno R, Ngameni B, Tsafack AM, Ngandeu F, Fotso GW, Bezabih M, Etoa FX, Ngadjui BT, Abegaz BM, Beng VP. Antimicrobial activity of the methanolic extracts and compounds from Treculia obovoidea (Moraceae). J Ethnopharmacol 2007; 112: 531-536

23 Adjanohoun JE, Aboubakar N, Dramane K, Ebot ME, Ekpere JA, Enow-Orock EG, Focho D, Gbile ZE, Kamanyi A, Kamsu Ko J, Keita A, Mbenkum T, Mbi CN, Mbiele AL, Mbome IL, Miburu NK, Nancy WL, Nkongmeneck B, Satabie B, Sofowora A, Tamze V, Wirmum CK. Traditional Medicine and Pharmacopoeia: Contribution to ethnopharmacological and floristic Studies in Cameroon. Lago: OAU/STRC; 1996: 299

24 Tekeu JC. Rapport sur la Pratique des Etudes d'Impact Environnemental (EIE) au Cameroun. Préparé pour la Commission Economique pour l'Afrique des Nations Unies. Available at: http://www.uneca.org/sdd/ documents/ReportEIACameroonFinal.pdf. 2004. Accessed on August 03, 2008
25 Aligiannis N, Kalpotzakis E, Mitaku S, Chinou IB. Composition and antimicrobial activity of the essential oils of two Origanum species. J Agric Food Chem 2001; 40: 4168-4170

26 Jimenez-Arellanes A, Meckes M, Ramirez R, Torres J, Luna-Herrera J. Activity against multidrug-resistant Mycobacterium tuberculosis in Mexican plants used to treat respiratory diseases. Phytother Res 2003; 17: 903-908

27 Tosun F, Akyüz KC, Sener B, Vural M, Palittapongarnpim P. Antimycobacterial screening of some Turkish plants. J Ethnopharmacol 2004; 95: 273-275

28 Molina-Salinas GM, Ramos-Guerra MC, Vargas-Villarreal J, Mata-Cardenas BD, Becerril-Montes P, Said-Fernández S. Bactericidal activity of organic extracts from Flourensia cernua DC against strains of Mycobacterium tuberculosis. Arch Med Res 2006; 37: 45-49

29 Borges-Argáez R, Canche-Chay CI, Peña-Rodríguez LM, Salvador SaidFernández S, Molina-Salinas GM. Antimicrobial activity of Diospyros anisandra. Fitoterapia 2007; 78: 370-372

30 Rios JL, Recio MC. Medicinal plants and antimicrobial activity. J Ethnopharmacol 2005; 100: 80-84

31 Cos P, Maes L, Sindambiwe JB, Vlietinck AJ, Berghe VD. Bioassays for antibacterial and antifungal activities. Biological screening of plant constituents. Training manual. Trieste: UNIDO-ICS (United Nations Industrial Development Organization and the International Centre for Science and High Technology); 2006: 19-28

32 De las Heras B, Rodríguez B, Boscá L, Villar AM. Terpenoids: sources, structure elucidation and therapeutic potential in inflammation. Curr Top Med Chem 2003; 3: 171-185

33 Fujioka T, Kashiwada Y. Anti-AIDS agents. 11. Betulinic acid and platanic acid as anti-HIV principles from Syzigium claviflorum, and the anti-HIV activity of structurally related triterpenoids. J Nat Prod 1994; 57: 243-247

34 Ragasa CY, Ha HK, Hasika M, Maridable JB, Gaspillo PD, Rideout JA. Antimicrobial and cytotoxic terpenoids from Cymbopogon citratus Stapf Philipp Sci 2008; 45: 111-122

35 Kadota S, Basnet P, Ishii E, Tamura T, Namba T. Antibacterial activity of trichorabdal from Rabdosia trichocarpa against Helicobacter pylori. Zentralbl Bakteriol 1997; 286: 63-67

36 Prabuseenivasan S, Jayakumar M, Ignacimuthu S. In vitro antibacterial activity of some plant essential oils. BMC Complement Altern Med 2006; 6: 39

37 John AJ, Karunakran VP, George V. Chemical composition an antibacterial activity of Neolitsea foliosa (Nees) Gamble var. caesia (Meisner) Gamble. J Essent Oil Res 2007; 19: 498-500

38 Nasimul Islam AKM, Abas Ali M, Sayeed A, Syed M, Salam A. An antibacterial terpenoid from Caesalpinia pulcherrina Swartz.: its characterization, antimicrobial and cytotoxic activities. Asian J Plant Sci 2003; 2: 1162-1665

39 Kuete V, Wabo GF, Ngameni B, Tsafack AM, Metuno R, Etoa FX, Ngadju $B T$, Beng VP. Antimicrobial activity of the methanolic extract and compounds from the stem bark of Irvingia gabonensis (Ixonanthaceae). J Ethnopharmacol 2007; 114: 54-60

40 Kuete V, Nguemeving JR, Beng VP, Azebaze AGB, Etoa FX, Meyer M, Bodo B, Nkengfack $A E$. Antimicrobial activity of the methanolic extracts and compounds from Vismia laurentii De Wild (Guttiferae). J Ethnopharmacol 2007; 109: 372-379

41 Kuete V, Wansi JD, Mbaveng AT, Kana Sop MM, Tadjong AT, Beng VP, Etoa FX, Wandji J, Meyer JJM, Lall N. Antimicrobial activity of the methanolic extract and compounds from Teclea afzelii (Rutaceae). S Afr J Bot 2008; 74: 572-576

42 Kuete V, Simo IK, Beng VP, Bigoga JD, Kapguep RN, Etoa FX, Ngadjui BT. Antimicrobial activity of the methanolic extract, fractions and four flavonoids from the twigs of Dorstenia angusticornis Engl. (Moraceae). J Ethnopharmacol 2007; 112: 271-277

43 Kuete V, Ngameni B, Tsafack AM, Ambassa IK, Simo P, Roy R, Bezabih M, Etoa FX, Ngadjui BT, Abegaz BM, Beng VP. Antimicrobial activity of the extract from the twigs of Dorstenia elliptica (Moraceae). Pharmacologyonline 2007; 1: 573-580

44 Kuete V, Ngameni B, Fotso Simo CC, Kengap Tankeu R, Tchaleu Ngadjui B, Meyer JJM, Lall N, Kuiate JR. Antimicrobial activity of the crude extracts and compounds from Ficus chlamydocarpa and Ficus cordata (Moraceae). J Ethnopharmacol 2008; 120: 17-24

45 Kuete V, Fozing DC, Kapche WFGD, Mbaveng AT, Kuiate JR, Ngadjui BT, Abegaz BM. Antimicrobial activity of the methanolic extract and compounds from Morus mesozygia stem bark. J Ethnopharmacol 2009; 124: 551-555 
46 Kapche GDWF, Fozing CD, Donfack JH, Fotso FW, Amadou D, Tchana AN, Bezabih M, Moundipa PF, Ngadjui BT, Abegaz BM. Moracin Q-U, new antioxidant prenylated arylbenzofuran derivatives from Morus mesosygia. Phytochemistry 2009; 70: 216-221

47 Aslam SN, Stevenson PC, Kokubun T, Hall DR. Antibacterial and antifungal activity of cicerfuran and related 2-arylbenzofurans and stilbenes. Microbiol Res 2009; 164: 191-195

48 Kuete V, Eyong KO, Beng VP, Folefoc GN, Hussain H, Krohn K, Nkengfack $A E$, Saeftel M, Sarite SR, Hoerauf A. Antimicrobial activity of the methanolic extract and compounds isolated from the stem bark of Newbouldia laevis Seem. (Bignoniaceae). Pharmazie 2007; 62: 552-556

49 Kuete V, Tangmouo JG, Marion Meyer JJ, Lall N. Diospyrone, crassiflorone, and plumbagin, three antimycobacterial and anti-gonorrheal naphthoquinones from two Diospyros species. Int J Antimicrob Agents 2009; 34: 322-325

50 Stern JL, Hagerman AE, Steinberg PD, Mason PK. Phorotannin protein interactions. J Chem Ecol 1996; 22: 1887-1889

51 Kuete V, Komguem J, Beng VP, Tangmouo JG, Meli AL, Etoa FX, Lontsi D. Antimicrobial components of the methanolic extract from the stem bark of Garcinia smeathmannii Oliver (Clusiaceae). S Afr J Bot 2007; 73: 347-354

52 Kuete V, Meli AL, Komguem J, Louh GN, Tangmouo JG, Lontsi D, Marion Meyer JJ, Lall N. Antimycobacterial, antibacterial and antifungal activities of the methanolic extract and compounds from Garcinia polyantha. Pharmacologyonline 2007; 3: 87-95

53 Mbaveng AT, Kuete V, Nguemeving JR, Krohn K, Nkengfack AE, Meyer JJM, Lall N. Antimicrobial activity of the extracts and compounds from Vismia guineensis (Guttiferae). AJTM 2008; 3: 211-223

54 Nguemeving JR, Azebaze AGB, Kuete V, Carly NNE, Beng VP, Meyer M, Bodo $B$, Nkengfack $A E$. Laurentixanthones $A$ and $B$, antimicrobial xanthones from Vismia laurentii. Phytochemistry 2006; 67: 1341-1346

55 Azebaze AGB, Ouahouo BMW, Vardamides JC, Valentin A, Kuete V, Acebey $L$, Beng VP, Nkengfack AE, Meyer M. Allaxanthones A and B, antimicrobial xanthones from Allablackia gabonensis. Nat Prod Res 2008; 4: 333341

56 Nkengfack AE, Mkounga P, Meyer M, Omum ZT, Bodo B. Globulixanthones C, D and E: three prenylated xanthones with antimicrobial properties from the root bark of Symphonia globulifera. Phytochemistry 2002; 61: 181-187

57 Berkada B. Preliminary report on warfarin for the treatment of Herpes simplex. J Ir Coll Physicians Surg 1978; 22: 56

58 Hoult JRS, Paya M. Pharmacological and biochemical actions of simple coumarins: natural products with therapeutic potential. Gen Pharmacol 1996; 27: 713-722

59 Casley-Smith JR, Casley-Smith JR. Coumarin in the treatment of lymphoedema and other high-protein oedemas. In: O'Kennedy R, Thornes RD, editors. Coumarins: biology, applications and mode of action. New York: John Wiley and Sons, Inc.; 1997: 348

60 De Souza SM, Monache FD, Smania Jr A. Antibacterial activity of coumarins. Z Naturforsch C 2005; 60: 693-700

61 Razavi SM, Imanzadeh G, Davari M. Coumarins from Zosima absinthifolia seeds, with allelopatic effects. EurAsia J BioSci 2010; 4: 17-22

62 Kuete V, Metuno R, Ngameni B, Tsafack AM, Ngandeu F, Fotso GW, Bezabih M, Etoa FX, Ngadjui BT, Abegaz BM, Beng VP. Antimicrobial activity of the methanolic extracts and compounds from Treculia obovoidea (Moraceae). S Afr J Bot 2008; 74: 111-115

63 Cos P, Maes L, Vlietinck A, Pieters L. Plant-derived leading compounds for chemotherapy of human immunodefiency virus (HIV) infection an update (1998-2007). Planta Med 2008; 74: 1323-1337

64 Hallock YF, Manfredi K, Dai J, Cardellina II JH, Gulakowski RJ, McMahon JB, Schäffer M, Stahl M, Gulden KP, Bringmann G, François G, Boyd MR. Michellamines D-F, new HIV-inhibitory dimeric naphthylisoquinoline alkaloids, and korupensamine E, a new antimalarial monomer, from Ancistrocladus korupensis. J Nat Prod 1997; 60: 677-683

65 White EL, Chao WR, Ross LJ, Borhani DW, Hobbs PD, Upender V, Dawson MI. Michellamine alkaloids inhibit protein kinase C. Arch Biochem Biophys 1999; 365: 25-30

66 Karpas A, Fleet GWJ, Dwek RA, Petursson S, Namgoong SK, Ramsden NG, Jacob GS, Rqdemqcher TW. Aminosugar derivatives as potential antihuman immunodeficiency virus agents. Proc Natl Acad Sci USA 1988; 85: 9229-9233

67 Watson AA, Fleet GWJ, Asano N, Molyneux RJ, Nash RJ. Polyhydroxylated alkaloids - natural occurrence and therapeutic applications. Phytochemistry 2001; 56: 265-295
68 Duan H, Takaishi Y, Imakura Y, Jia Y, Li D, Cosentino M, Lee KH. Sesquiterpene alkaloids from Tripterygium hypoglaucum and Tripterygium wilfordii: a new class of potent anti-HIV agents. J Nat Prod 2000; 63: 357-361

69 Ishida J, Wang HK, Oyama M, Cosentino ML, Hu CQ, Lee KH. Anti-AIDS agents. 46. Anti-HIV activity of harman, an anti-HIV principle from Symplocos setchuensis, and its derivatives. J Nat Prod 2001; 64: 958960

70 Meragelman KM, McKee TC, Boyd MR. Siamenol, a new carbazole alkaloid from Murraya siamensis. J Nat Prod 2000; 63: 427-428

71 Jones Jr SB, Luchsinger AE. Plant systematics. New York: McGraw-Hill Book Co.; 1986

72 Atta-ur-Rahman, Choudhary MI. Diterpenoid and steroidal alkaloids. Nat Prod Rep 1995; 12: 361-379

$73 \mathrm{Yi} \mathrm{ZB,} \mathrm{Yu} \mathrm{Y,} \mathrm{Liang} \mathrm{YZ,} \mathrm{Zeng} \mathrm{B.} \mathrm{Evaluation} \mathrm{of} \mathrm{the} \mathrm{antimicrobial} \mathrm{mode} \mathrm{of}$ berberine by LC/ESI-MS combined with principal component analysis. J Pharm Biomed Anal 2007; 44: 301-304

74 Bruneton J. Pharmacognosie: Phytochimie, Plantes medicinales, 3rd edition. Paris: Tec \& Doc; 1999: 263-309

75 Ngounou FN, Manfouo RN, Tapondjou LA, Lontsi D, Kuete V, Penlap V, Etoa FX, Dubois MAL, Sondengam BL. Antimicrobial diterpenoid alkaloids from Erythrophleum suaveolens (Guill. \& Perr.) Brenan. Bull Chem Soc Ethiop 2005; 19: 221-226

76 Kuete $V$. Evaluation des propriétés antimicrobiennes de deux plantes médicinales Camerounaises utilises dans le traitement des maladies infectieuses: Solanum trvum (Solanaceae) et Tabernaemontana crassa (Apocynaceae) [dissertation]. Yaoundé: Université de Yaoundé I; 2005

77 Tatsadjieu LN, Essia Ngang JJ, Ngassoum MB, Etoa FX. Antibacterial and antifungal activity of Xylopia aethiopica, Monodora myristica, Zanthoxylum xanthoxyloïdes and Zanthoxylum leprieurii from Cameroon. Fitoterapia 2003; 74: 469-472

78 Burkill HM. The Useful plants of west tropical Africa, Vol. 1. Kew: Royal Botanic Gardens; 1985: 186-187

79 Wagner WL, Herbst DR, Sohmer SH. Manual of the Flowering Plants of Hawaii: revised ed. Honolulu: University of Hawaii Press; 1999: 312

80 Ngo Teke G, Kuiate JR, Ngouateu OB, Gatsing D. Antidiarrhoeal and antimicrobial activities of Emilia coccinea (Sims) G. Don extracts. J Ethnopharmacol 2007; 112: 278-283

81 Ndom JC, Mbafor JT, Wansi JD, Kamdem AW, Meva'a LM, Vardamides JC, Toukam F, Pegyemb D, Ngando TM, Laatsch H, Fomum ZT. Sesquiterpene lactones from Crepis cameroonica (Asteraceae). Nat Prod Res 2006; 20 : 435-442

82 Eyong KO, Folefoc GN, Kuete V, Beng VP, Krohn K, Hussain H, Nkengfack $A E$, Saeftel M, Sarite SR, Hoerauf A. Newbouldiaquinone A: a naphthoquinone-anthraquinone ether coupled pigment, as a potential antimicrobial and antimalarial agent from Newbouldia laevis. Phytochemistry 2006; 67: 605-609

83 Eyong OK, Krohn K, Hussain H, Folefoc NG, Nkengfack AE, Schulz B, Hu Q. Newboudiaquinone and newboudiamide: A naphthoquinone-anthraquinone couple pigment and a new ceramide from Newboudia laevis. Chem Pharm Bull 2005; 53: 616-619

84 Lenta BN, Weniger B, Antheaume C, Noungoue DT, Ngouela S, Assob JCN, Vonthron-Sénécheau C, Fokou PA, Devkota KP, Tsamo E, Sewald N. Anthraquinones from the stem bark of Stereospermum zenkeri with antimicrobial activity. Phytochemistry 2007; 68: 1595-1599

85 Hutchinson J, Dalziel JM. Flora of West Tropical Africa, 2nd edition. London: Crown Agents; 1958

86 Tangmouo JG, Lontsi D, Ngounou FN, Kuete V, Meli AL, Manfouo RN, Kamdem HW, Tane P, Beng VP, Sondengam BL, Connolly JD. Diospyrone, a new coumarinylbinaphthoquinone from Diospyros canaliculata (Ebenaceae): structure and antimicrobial activity. Bull Chem Soc Ethiop 2005; 19: 81-88

87 Tangmouo JG, Meli AL, Komguem J, Kuete V, Ngounou FN, Lontsi D, Beng VP, Choudhary MI, Sondengam BL. Crassiflorone, a new naphthoquinone from Diospyros crassiflora (Hien). Tetraedron Lett 2006; 47: 3067-3070

88 Dzoyem JP, Tangmouo JG, Lontsi D, Etoa FX, Lohoue PJ. In vitro antifungal activity of extract and plumbagin from the stem bark of Diospyros crassiflora Hiern (Ebenaceae). Phytother Res 2007; 21: 671-674

89 Dimo T, Laure NE, Benoit NT, Anatole AGB, Paul AA, Emmanuel TV, Pierre $K$. Antinociceptive and anti-inflammatory effects of the ethyl acetate stem bark extract of Bridelia scleroneura (Euphorbiaceae). Inflammopharmacology 2006; 14: 42-47 
90 Ngueyem TA, Brusotti G, Marrubini G, Grisoli P, Dacarro C, Vidari G, Finzi PV, Caccialanza G. Validation of use of a traditional remedy from Bridelia grandis (Pierre ex Hutch) stem bark against oral Streptococci. J Ethnopharmacol 2008; 120: 13-16

91 Dalziel JM. The useful plants of west tropical Africa. London: The Crown Agents for the Colonies; 1937: 140-141

92 Talla E, Djamen D, Djouldé DR, Tatsadjeu L, Tantoh D, Mbafor JT, Fomum $Z T$. Antimicrobial activity of Bridelia ferruginea leaves extracts. Fitoterapia 2002; 73: 343-345

93 Kamgang R, Pouokam Kamgne EV, Fonkoua MC, Beng VP, Biwolé SM. Activities of aqueous extracts of Mallotus oppositifolium on Shigella dysenteriae A1-induced diarrhoea in rats. Clin Exp Pharm Physiol 2006; 33: 89-94

94 Awouafack MD, Kouam FS, Hussain H, Ngamga D, Tane P, Schulz B, Green IR, Krohn K. Antimicrobial prenylated dihydrochalcones from Eriosema glomerata. Planta Med 2008; 74: 50-54

95 Ouahouo BMW, Azebaze AGB, Meyer M, Bodo B, Fomum ZT, Nkengfack $A E$. Cytotoxic and antimicrobial coumarins from Mammea africana. Ann Trop Med Parasitol 2004; 98: 733-739

96 Yimdjo MC, Azebaze AGB, Nkengfack AE, Meyer AM, Bodo B, Fomum ZT. Antimicrobial and cytotoxic agents from Calophyllum inophyllum. Phytochemistry 2004; 65: 2789-2795

97 Akoachere JF, Ndip RN, Chenwi EB, Ndip LM, Njock TE, Anong DN. Antibacterial effect of Zingiber officinale and Garcinia kola on respiratory tract pathogens. East Afr Med J 2002; 79: 588-592

98 Ngoupayo J, Tabopda TK, Shaiq Ali M. Antimicrobial and immunomodulatory properties of prenylated xanthones from twigs of Garcinia staudtii. Bioorg Med Chem 2009; 17: 5688-5695

99 Aubreville A. Flore forestière soudano-guinéenne A.O.F. Cameroun-A. E.F. Paris: Société d'Edition Géographique Maritime et Coloniales; 1950: $148-150$

100 Ngouela S, Ndjakou BL, Tchamo DN, Zelefack F, Tsamo E, Connolly JD. A prenylated xanthone with antimicrobial activity from the seeds of Symphonia globulifera. Nat Prod Res 2002; 19: 23-27

101 Menan H, Banzouzi JT, Hocquette A, Pelissier Y, Blache Y, Kone M, Mallie $M$, Ake Assi L, Valentin A. Antiplasmodial activity and cytotoxicity of plants used in West African traditional medicine for the treatment of malaria. J Ethnopharmacol 2006; 105: 131-136

102 Tamokou JDD, Tala MF, Wabo HK, Kuiate JR, Tane P. Antimicrobial activities of methanol extract and compounds from stem bark of Vismia rubescens. J Ethnopharmacol 2009; 124: 571-575

103 Berhaut J. Flore illustrée du Sénégal, Dicotylédones, Linacées à Nymphéacées, Tome VI. Dakar: Gouvernement du Sénégal, Ministère du Développement Rural et de l'Hydraulique, Direction des eaux et Forêts; 1979: 466-481

104 Adamson I, Okafor C, Abu-Bakare A. A supplement of Dikanut (Irvingia gabonensis) improves treatment of type II diabetics. West Afr J Med 1990; 9: 108-115

105 Okolo C, Johnson P, Abdulrahman F, Abdu-Aguye I, Hussaini I. Analgesic effect of Irvingia gabonensis stem bark extract. J Ethnopharmacol 1995; 45: 125-129

106 Adamson I, Okafor C, Abu-Bakare A. Erythrocyte membrane ATPases in diabetes: effect of Dikanut (Irvingia gabonensis). Enzyme 1986; 36: 212-215

107 Ngondi J, Oben J, Minka S. The effect of Irvingia gabonensis seeds on body weight and blood lipids of obese subject in Cameroon. Lipids Health Dis 2005; 4: 12

108 Ngassoum MB, Essia-Ngang JJ, Tatsadjieu LN, Jirovetz L, Buchbauer G, Adjoudjia O. Antimicrobial study of essential oils of Ocimum gratissimum leaves and Zanthoxylum xanthoxyloides fruits from Cameroon. Fitoterapia 2003; 74: 284-287

109 Nguefack J, Lekagne Dongmo JB, Dakole CD, Leth V, Vismer HF, Torp J, Guemdjom EFN, Mbeffo M, Tamgue O, Fotio D, Amvam Zollo PH, Nkengfack $A E$. Food preservative potential of essential oils and fractions from Cymbopogon citratus, Ocimum gratissimum and Thymus vulgaris against mycotoxigenic fungi. Int J Food Microbiol 2009; 131: 151-156

110 Chouna JR, Nkeng-Efouet PA, Lenta BN, Devkota PK, Neumann B, Stammler HG, Kimbu SF, Sewald N. Antibacterial endiandric acid deriv- atives from Beilschmiedia anacardioides. Phytochemistry 2009; 70: 684-688

111 Thomas DW, Thomas JM, Bromely WA, Mbenkum FT. Korup ethnobotany survey: WWF Survey. 1989: A31

112 Abegaz BM, Ngadjui TB, Dongo E, Tamboue H. Prenylated chalcones and flavones from the leaves of Dorstenia kameruniana. Phytochemistry 1998; 49: 1147-1150

113 Tsopmo A, Tene M, Kamnaing P, Ayafor JF, Sterner A. A new Diels-Alder type adduct flavonoids from $D$. barteri. J Nat Prod 1999; 62: 1432 1434

114 Bouquet A. Féticheurs et médecines traditionnelles du Congo Brazzaville. Paris: Orstom; 1969: 178-202

115 Kuete V, Nana F, Ngameni B, Mbaveng AT, Keumedjio F, Ngadjui BT. Antimicrobial activity of the crude extract, fractions and compounds from stem bark of Ficus ovata (Moraceae). J Ethnopharmacol 2009; 124: $556-561$

116 Noumi E, Dibakto TW. Medicinal plants used for peptic ulcer in the Bangangté region, western Cameroon. Fitoterapia 2002; 71: 406-512

117 Bokesch HR, Charan RD, Merangelman KM, Beutler JA, Gardella $R$, O'keefe BR, Mekee TC, Memahon JB. Isolation and characterization of anti-HIV peptides from Dorstenia contrajerva and Treculia obovoidea. FEBS Lett 2004; 567: 287-290

118 Kouam SF, Yapna DB, Krohn K, Ngadjui BT, Ngoupayo J, Choudhary MI, Schultz B. Antimicrobial prenylated anthracene derivatives from the leaves of Harungana madagascariensis. J Nat Prod 2007; 70: 600-603

119 Vardamides JC, Sielenou VT, Ndemangou B, Nkengfack AE, Fomum ZT, Poumale HMP, Laatsch H. Diterpenoids from Turraeanthus mannii. Planta Med 2007; 5: 491-495

120 Njike NE, Watch P, Nguelefack T, Kamanyi A. Hypoglycaemic activity of the leaves extracts of Bersama engleriana in rats. Afr J Trad CAM 2005; 2: 215-221

121 Watcho P, Makemdjio A, Nguelefack BT, Kamanyi A. Sexual stimulation effects of the aqueous and methanolic extracts from the leaves of Bersama engleriana in adult male rats. Pharmacologyonline 2007; 1 : 464-476

122 Kuete V, Tsafack AM, Tsafack M, Beng VP, Etoa FX, Nkengfack AE, Meyer $J J M$, Lall N. Antitumor, antioxidant and antimicrobial activities of Bersama engleriana (Melianthaceae). J Ethnopharmacol 2008; 115: 494501

123 Zintchem AA, Ngono Bikobo D, Théodore Atchadé AT, Ngo Mbing J, Gangoue-Pieboji J, Ghogomu Tih R, Blond A, Pegnyemb DE, Bodo B. Nitrile glucosides and serotobenine from Campylospermum glaucum and Ouratea turnarea. Phytochemistry 2008; 69: 2209-2213

124 Pegnyemb DE, Ngo Mbing J, Atchade AT, Ghogomu Tih R, Sondengam BL, Blond A, Bodo B. Antimicrobial biflavonoids from the aerial parts of Ouratea sulcata. Phytochemistry 2005; 66: 1922-1926

125 Irvine RF. Woody plant of Ghana. London: Oxford University Press; 1961: 223-226; 480-481

126 Fokou PA, Stammler HG, Neumann B, Huber T, Lontsi D, Kemami Wangun HV, Sewald N. Triterpenes from Maesopsis eminii. J Nat Prod 2004; 67: 2124-2126

127 Adnan JA, Mohammad SA, Ilias M, Assad AA, Herman PP. Furoquinoline alkaloids from Teclea nobilis. Phytochemistry 2003; 66: 1405-1411

128 Wansi JD, Wandji J, Waffo AF, Ngeufa HE, Ndom JC, Fotso S, Maskey RP, Njamen D, Fomum TZ, Laatsch H. Alkaloids from Oriciopsis glaberrima Engl. (Rutaceae). Phytochemistry 2006; 67: 475-480

129 Kuete V, Tangmouo JG, Beng VP, Ngounou MF, Lontsi D. Antimicrobial activity of Tridesmostemon omphalocarpoides Engl. Sapotaceae. J Ethnopharmacol 2006; 104: 5-11

130 Megne $B C$. Contribution à l'étude des plantes médicinales du Camerounieuses: Inventaire de quelques plantes utilisées dans le traitement des MST dans la région de Dschang (Ouest-Cameroun) [Mémoire de Maîtrise]. Dschang: Université de Dschang; 1998

131 Arthan D, Svasti J, Kittakoop P, Pittayakhachonwut D, Tanticharoen M, Thebtaranonth $Y$. Antiviral isoflavonoidsulphate and steroidal glycosides from the fruits of Solanum torvum. Phytochemistry 2002; 59: 459-464

132 Chah KF, Muko KN, Oboegbulem SI. Antimicrobial activity of methanolic extract of Solanum torvum fruit. Fitoterapia 2000; 71: 187-189 